\title{
Less Information, Similar Performance: Comparing Machine Learning-Based Time Series of Wind Power Generation to Renewables.ninja
}

\author{
Johann Baumgartner ${ }^{1, *}$, Katharina Gruber ${ }^{1}$, , Sofia G. Simoes ${ }^{2}$, Yves-Marie Saint-Drenan ${ }^{3}$ and \\ Johannes Schmidt ${ }^{1}$ \\ 1 Institute for Sustainable Economic Development, University of Natural Resources and Life Sciences, \\ 1180 Vienna, Austria; katharina.gruber@boku.ac.at (K.G.); johannes.schmidt@boku.ac.at (J.S.) \\ 2 LNEG-The National Laboratory for Energy and Geology, Resource Economics Unit, 1649-038 Lisbon, \\ Portugal; sofia.simoes@lneg.pt \\ 3 MINES ParisTech, PSL Research University, O.I.E. Centre Observation, Impacts, Energy, \\ 06904 Sophia Antipolis, France; yves-marie.saint-drenan@mines-paristech.fr \\ * Correspondence: johann.baumgartner@boku.ac.at
}

Received: 24 February 2020; Accepted: 30 April 2020; Published: 5 May 2020

\begin{abstract}
Driven by climatic processes, wind power generation is inherently variable. Long-term simulated wind power time series are therefore an essential component for understanding the temporal availability of wind power and its integration into future renewable energy systems. In the recent past, mainly power curve-based models such as Renewables.ninja (RN) have been used for deriving synthetic time series for wind power generation, despite their need for accurate location information and bias correction, as well as their insufficient replication of extreme events and short-term power ramps. In this paper, we assessed how time series generated by machine learning models (MLMs) compare to RN in terms of their ability to replicate the characteristics of observed nationally aggregated wind power generation for Germany. Hence, we applied neural networks to one wind speed input dataset derived from MERRA2 reanalysis with no location information and two with additional location information. The resulting time series and RN time series were compared with actual generation. All MLM time series feature an equal or even better time series quality than $\mathrm{RN}$, depending on the characteristics considered. We conclude that MLM models show a similar performance to $\mathrm{RN}$, even when information on turbine locations and turbine types is unavailable.
\end{abstract}

Keywords: wind power simulation; wind power time series; reanalysis; machine learning

\section{Introduction}

Globally, the installed wind power capacity has increased more than six-fold within eleven years, from 93.9 GW in 2007 to 591.5.8 GW in 2018. As of 2018, nearly one tenth of the global installed wind power capacity was located in Germany, where the installed wind power capacity increased from 22.2 GW in 2007 to $53.2 \mathrm{GW}$ in 2018. This increase resulted in a power generation of $111.5 \mathrm{TWh}$ in 2018, corresponding to $21 \%$ of the electricity demand in Germany [1-4].

Due to this significant expansion, the spatial and temporal availability of climate-dependent wind resources increasingly affects the whole power system. Consequently, assessments of the electricity system's vulnerability to extreme climate events and climate change by means of power system models provide vital insights into how future electricity systems should be structured to mitigate supply scarcity and power outages [5-7]. Therefore, accurate multi-year generation time series (i.e., multiple years of temporally highly-resolved values) are used as input data for power system models (e.g., $[8,9])$ as well as energy system models (e.g., [10-12]), for quantifying the system's resilience to climate events. 
This is increasingly important when an even higher market penetration of renewables is taken into account [13].

In the recent past, mainly power curve-based models based on reanalysis climate data sets [13-19] have been used for deriving multi-year time series. These models have been able to reproduce wind power generation time series sufficiently well in terms of error metrics, and distributional and seasonal characteristics. However, these models also feature possible drawbacks, such as the high data requirements for model setup (i.e., wind turbine locations, turbine specifications, and commissioning dates) and the need for separate work steps for bias correction and the replication of wake effects (e.g., power curve smoothing) $[13,18,19]$. In particular, well known shortcomings of reanalysis data, i.e., a significant mean bias in wind speeds, have to be overcome by the models via bias correction [19], which relies on the availability of historical wind power generation time series or independent sources of wind speed data, such as local wind speed measurements. Another downside of reanalysis-based time series generated by power curve-based models has been their insufficient replication of extreme generation events and short-term power ramps $[13,15,18]$. This can only partly be attributed to the methodology, as the underlying reanalysis data sources do not sufficiently capture extreme situations [18]. The accurate replication of power generation extremes and potential generation changes within short- $(1 \mathrm{~h})$, mid- $(3$ and $6 \mathrm{~h})$, and long-term $(12 \mathrm{~h})$ timeframes, however, would be of high value for power system models.

As real-generation time series are necessary for bias-correction or validation anyhow, instead of using power curve-based models, machine learning (ML) models can be applied to derive synthetic time series from climate data for time periods where no observed generation is available.

In particular, neural networks are a promising approach. They can fit arbitrary, non-linear functions as they are universal function approximators [20]. The need for a correction of systematic biases as a separate work step and the need for information on accurate turbine locations and other specifications can therefore possibly be overcome when using machine learning wind power generation models. This decreases the effort required when generating time series of wind power electricity generation, as gathering accurate information on turbine locations can be time-consuming or even impossible for some countries. Additionally, while machine learning (ML) models based on the same underlying climatic data cannot be expected to fully solve the problem of the correct representation of real wind power variability, they may increase the quality of results in terms of extreme values by learning spatio-temporal relationships between the climatic input and (extreme) values for the output.

In this paper, we consequently assessed whether machine learning (ML) models are equally or even better suited than Renewables.ninja (RN)—a cutting-edge power curve-based model time series [13] - for replicating the distributional, seasonal, extreme value, and power ramp characteristics of actual wind power generation. Furthermore, we quantified how the quality of the ML modeled time series depends on the extent of information on wind turbine locations that the ML model receives. RN has been chosen as the comparison dataset because it is an openly available wind power dataset with a proven performance based on a state-of-the-art modeling approach. It has been found that RN wind power time series have more similarities to Transmission System Operator (TSO) data compared with EMHIRES [18] —a second cutting-edge power curve-based time series. For a limited number of years (2012-2014), the RN wind power time series showed a correlation of 0.98 compared with TSO data [21]. A multitude of research projects have successfully used the RN wind power dataset as a data source for assessing balancing of the wind power output via the spatial deployment of wind power, in order to understand the impacts of the inter-annual variability of intermittent renewables on the European power system and to implement decision support tools for managing flexibility in power systems with high shares of renewables [22-24]. The successful application of ML models for short- and medium-term predictions of wind power [25-28] provides reasonable arguments for using machine learning models (MLMs) for the purpose of generating synthetic wind power generation time series. However, MLMs have only been utilized to derive multi-year wind power generation time series or predictions on spatial dimensions larger than single power plant sites or wind farms 
within close spatial proximity [29-32]. A countrywide estimation of wind power time series by means of ML models for use in energy system models has not been conducted before, to the best of our knowledge. Additionally, it has not yet been assessed whether spatial information on the installed capacity is necessary to generate high-quality time series. This, in particular, addresses a significant knowledge gap as power curve-based models rely on temporally highly resolved information on wind farm locations and installed capacities. Location-specific information on installed capacities is often unavailable or not openly and freely available, so research is limited to using countrywide aggregated capacity data.

To address this research gap, in this paper, we trained a multilayer perceptron neural network to predict wind power generation from climate variables, using the Modern-Era Retrospective analysis for Research and Applications, Version 2 (MERRA2) as input. MERRA2 is a reanalysis dataset produced by the Global Modeling and Assimilation Office (GMAO) at National Aeronautics and Space Administration (NASA), with global coverage [33]. The MERRA2 dataset is also used by RN. We train three different ML models which differ in terms of the amount of information on turbine locations available to them. Thus, we are able to assess which spatial information on the installed capacity is necessary to generate high-quality wind power time series. Consequently, the three resulting time series are compared with time series generated by $\mathrm{RN}$ in terms of model error metrics and the representation of distributional, seasonal, extreme events, and power ramp characteristics for the period of 2012-2016.

\section{Materials and Methods}

In this study, we compared our ML-based time series to the RN data set [34]. The RN wind power model output, which we used for our analysis, was previously produced by another research group and is publicly available at [34]. Figure 1 shows a brief comparison of our modeling approach and RN. In contrast to the rather simple ML modeling process, $\mathrm{RN}$ has to perform five major steps to derive a wind power time series. Our ML modeling process trains a neural network by regressing a set of predictor variables (wind speed components $\mathrm{u}, \mathrm{v}$ and date dummies) on the response variable wind power generation (a) and uses the trained neural network to predict wind power generation by using a set of wind speed data from the same source but different time period and date dummy predictors (b) (Figure 1). A more detailed description of the ML approach can be found in Section 2.1.

In the RN approach, the following steps are taken (Figure 1):

(a) Wind speed data were acquired, and wind speed components, i.e., the $\mathrm{u}$ and $\mathrm{v}$ variables, were interpolated from the MERRA2 grid to the actual turbine locations using LOESS regression.

(b) Wind speeds were extrapolated to the corresponding turbine hub heights from the three height levels provided by MERRA2 $(2,10$, and $50 \mathrm{~m}$ above the ground) using the logarithmic wind profile.

(c) Wind speeds were converted to wind turbine power outputs by using manufacturers' power curves.

(d) An additional bias correction step for calibrating results against actual electricity generation was performed in the RN model as a post-processing step.

A detailed description of the modeling approach used by RN can be found in Staffell and Pfenninger [13]. In general, all power curve-based models follow a highly similar approach to RN.

Both our ML-based approach and RN used MERRA2 reanalysis as the data source for the 2, 10, and 50-m $\mathrm{u}$ and $\mathrm{v}$ wind speed components. We chose Germany as modeling location due to its highly developed wind turbine fleet, in addition to the sound availability of wind power generation data via the Open Power System Data (OPSD) platform [35]. The model time period (2010-2016) was chosen to fit the longest openly available coherent time series of hourly resolved wind power generation. The temporal resolution of observed and modeled generations was hourly. 
MLM

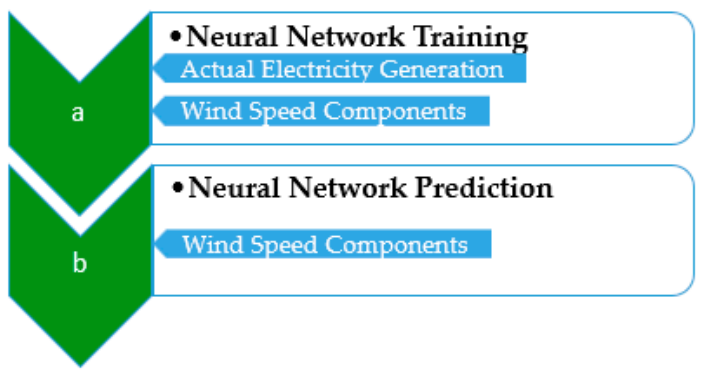

Input Data Flow

Modeling work step

\section{RN (Renewables.ninja)}

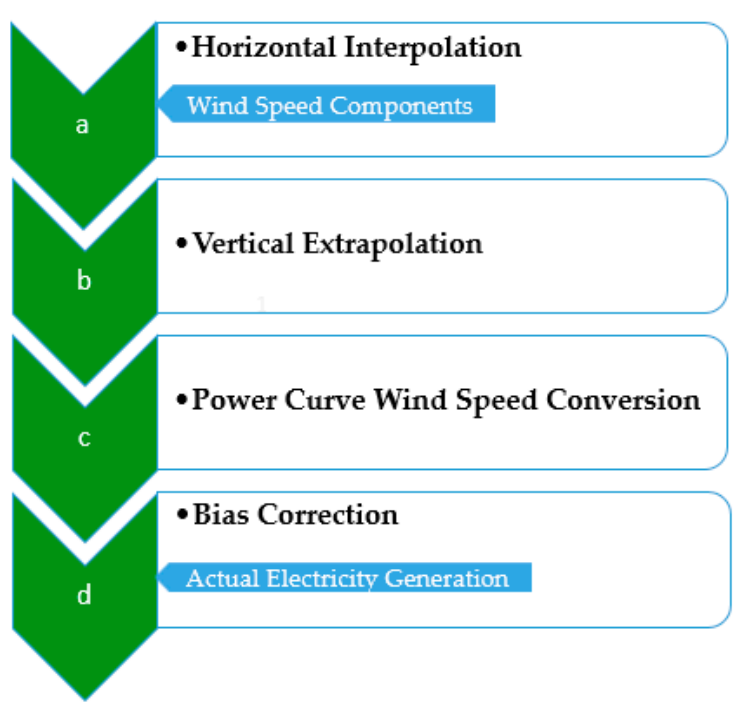

Figure 1. Comparison of the necessary modeling steps for deriving wind power output with the RN and the MLM approach indicated with letters a, b, c, d (Renewables.ninja abbreviated as RN, machine learning model abbreviated as MLM, own figure).

\subsection{Detailed Description of MLM}

Neural networks are commonly used algorithms for the short- and mid-term prediction of wind power generation [25,36-38]. In this study, we employed a multilayer perceptron neural network to regress climate variables and additional date dummies as predictor variables on the response variable, i.e., the actual wind power generation time series for Germany (Table 1), to generate long-term time series of wind power generation.

To quantify how the quality of the ML-modeled time series depends on the extent of information about wind turbine locations, three neural networks MLM1, MLM2, and MLM3 were trained with three different input datasets. These datasets differed with respect to the amount of wind speed component grid points used (see Section 2.2.1). The results of MLM2 and MLM3 do not differ strongly, results for MLM3 are therefore only shown in the Appendix A.

The preparatory steps for the MLM approach consist of acquiring the necessary climate input data and deriving date dummy variables for MLM1. For MLM1, all climate data grid points within a bounding box around Germany are used. For MLM2, only data grid points close to actual wind power turbine locations were selected and for MLM3 climate data grid points have been further reduced based on the amount of installed capacity (see Section 2.2.1). Subsequently, the input data were applied to train the model on observed generation and, using that trained model, to predict generation for a period not used in the training of the model. In the first step, the neural network model parameters, i.e., the weights in the network, are estimated using the input data from the training period (training dataset). To guarantee reproducibility of results, we had pre-set the seed for the random number generator. The trained neural network is consequently fed with the remaining set of input variables (prediction dataset) to compute the modeled electricity generation from wind power for the prediction period in terms of capacity factors. We used a neural network with one input layer with a node size equal to the number of input predictor variables, i.e., our dummy variables and wind speed components; three hidden layers of a user-defined size; and one output layer of size one, i.e., electricity generation from wind power, which was the predicted variable. The activation functions used in the three hidden layers are of the sigmoid type and the output layer activation is a linear function. The neural network weights (i.e., $\mathrm{w}_{\mathrm{ij}}$ and $\mathrm{w}_{\mathrm{jk}}$ ) were estimated by minimizing the error of the network output in comparison to observations. In the chosen neural network modeling framework, 
the Root Mean Square Error (RMSE) is the default error measure. Error backpropagation was used to minimize the error in an iterative process (Figure 2). For the hidden layers, we tested models with layer sizes of 60 and 80 nodes. Out of these two models, the model size which resulted in better correlation, normalized root mean square error (NRMSE), and normalized mean absolute error (NMAE) was chosen for further use.

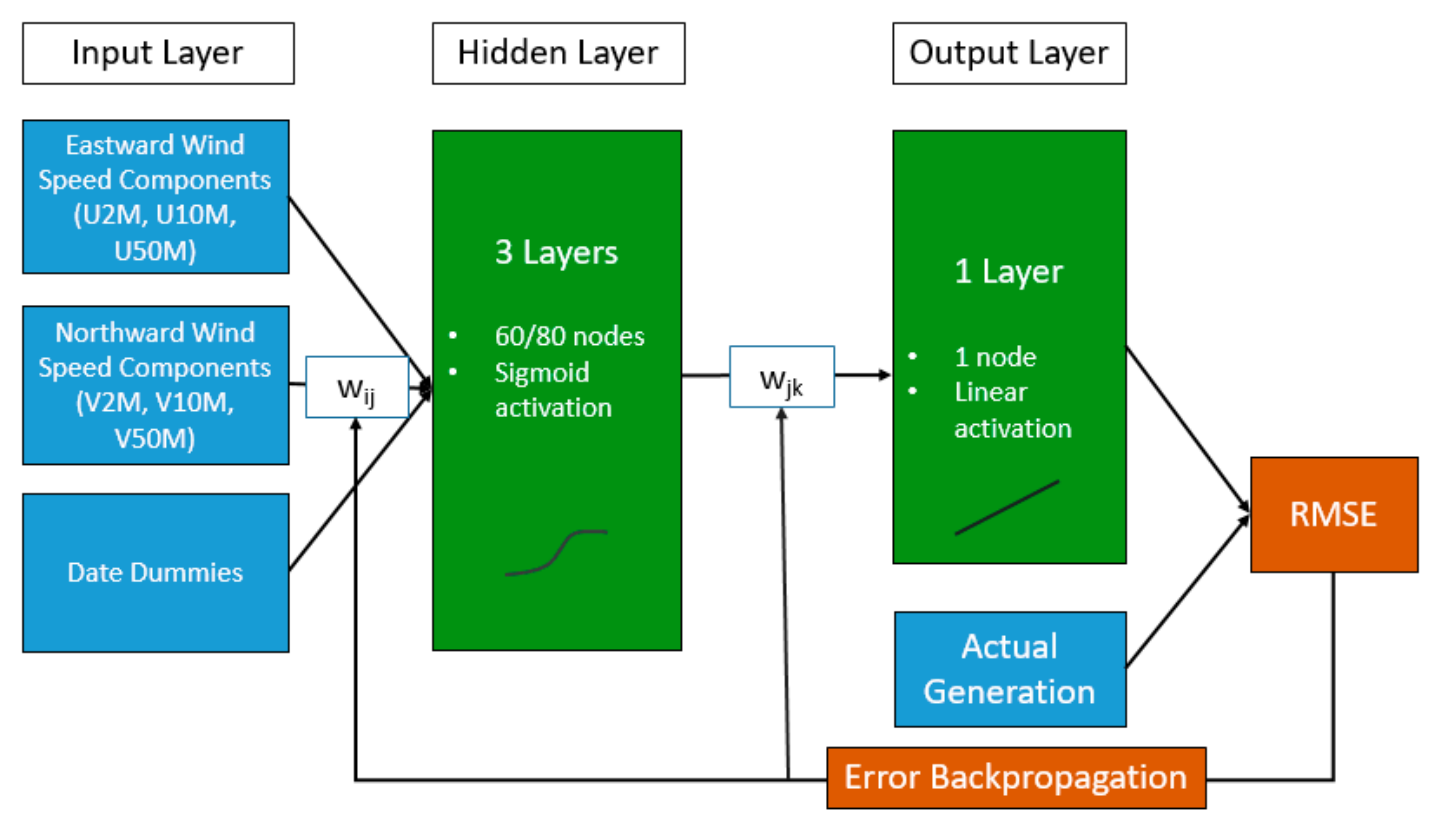

Figure 2. Schematic representation of the neural network model employed in the MLM approach.

In order to reduce the number of model training iterations needed to generate the seven-year time series and to provide a sufficient time period for model training, the training time period needs to be rearranged for every prediction period. This results in four training and prediction iterations illustrated as P1-P4 (Figure 3). For the prediction period of 2010 and 2011, the training period was set to 2012-2016. For the prediction period of 2012 and 2013, the years 2010-2011 and 2014-2016 were used for training, and similarly for all other periods. This resulted in a time series split between the training and testing period amounting to approximately 71 to $29 \%$ and ensured that every prediction was out-of-sample (Figure 3).

Function calls and computations used for our ML approach were executed according to the specifications in Bergmeir and Benítez [39] and the package documentation of the R-Package "caret" [40]. The model training part consisted of a call to a training function, which resulted in a neural network model calibrated for estimating nationally aggregated wind power electricity generation based on wind speed and date dummy predictor variables. This neural network model was then used to derive an hourly wind power electricity time series solely based on out-of-sample predictor input variables.

All computations and visualizations were conducted in RStudio (Version 1.1.423) with $R$ version "Microsoft R Open 3.4.3". The packages "tidyverse", "lubridate", and "ggplot2", and their corresponding dependencies were used for data handling and visualization. For model set up, training, and predictions, the package "caret" was used, which itself depends on the "RSNNS" package. Downloads and handling of MERRA-files were done with the R-package "MERRAbin" and its dependencies [41]. The source code for this methodological approach and for the validation is available in a GitHub repository [42]. The resulting ML-based time series have been made available as feather and CSV files on the hosting service Zenodo [43]. 


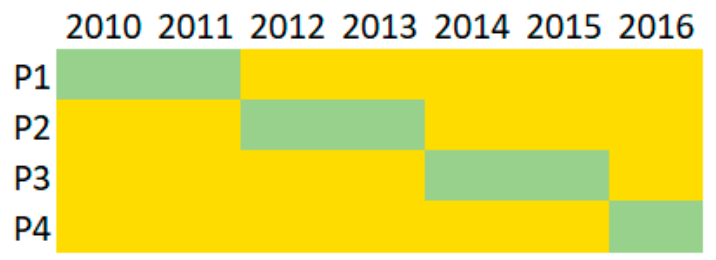

Training

Prediction

Figure 3. Training (yellow) and prediction (green) iterations P1-P4. In each iteration, model parameters are trained, and the associated prediction period is predicted with the neural network. All green periods are combined to form one 7 years timeseries.

\subsection{Data}

\subsubsection{Climate Input Data}

The present study is based on climate input variables from the global reanalysis data set MERRA2. This dataset was chosen to enable a comparison of our results and the time series from RN (ninja_europe_wind_v1.1-data package) [34], which uses the same data source. The climate input data used in the MLM are featured in the time-averaged single-level diagnostics subset "tavg1_2d_slv_Nx", whereby wind speed components U2M, V2M, U10M, V10M, U50M, and V50M, i.e., wind speeds at 2, 10 , and $50 \mathrm{~m}$ above the ground, were used.

These variables for all MERRA2 grid points within a bounding box (longitude from 5 to 15.625, latitude from 46 to 56) around Germany constitute the input data set for the first MLM-generated time series (MLM1), where no variable subsetting was performed and all data points were used. The input dataset for the time series MLM1 did not feature any location information at all, which meant that all wind speed grid points in a bounding box around Germany were used. The input dataset for MLM2 contained implicit information on turbine locations via climate variable subsetting, which corresponds to using only the four wind speed grid points closest to locations where wind turbines were actually installed by 1 January 2017. This resulted in the use of all grid points within Germany plus some adjacent points. For MLM3, the set of grid points was further reduced to contain only those closest to an installed capacity above the third quartile of the capacity distribution in Germany and here no neighboring grid points are included opposed to MLM2 (Figure 4). This subsetting is only possible when not only wind farm locations but also their installed capacities are known.

Therefore, the input datasets for MLM1, MLM2, and MLM3 only differ in the number of wind speed component values used (Table 1).

\subsubsection{Installed Capacity and Electricity Generation Data}

The observed electricity generation from OPSD [35] was used as the response variable for training the neural network for all MLM time series, as well as for assessing the quality of the modeled electricity generation time series.

These generation values were consequently converted to capacity factors $(\mathrm{CF})$ by dividing them by daily values of the installed wind power capacity from OPSD [35]. The locations and installed capacities of wind farms taken from OPSD [44] were additionally used to spatially subset climate data by extracting wind speed components closest to the wind farm locations for MLM2 and MLM3. The installed capacity time series does not explicitly feed into either of the MLMs for reasons of comparability ( $\mathrm{RN}$ time series are based on a single installed capacity value). Consequently, all variables were scaled by subtracting the mean and divided by the value range (minimum value subtracted from maximum value). Feature scaling is the standard procedure employed for reducing the computational effort in the training of neural networks. A list of all variables used in this study, in addition to their application, unit, size, and source, can be found in Table 1. 


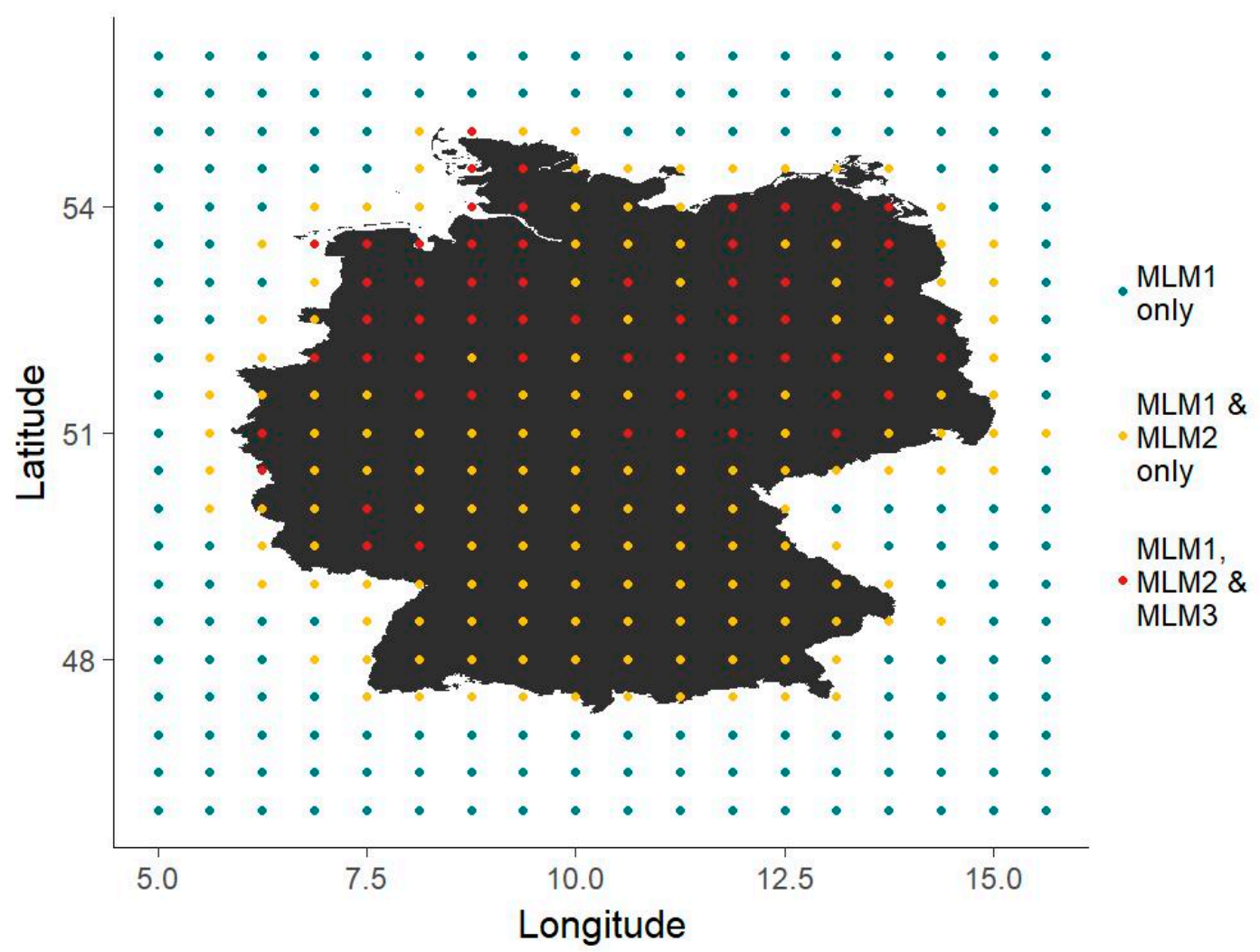

Figure 4. MERRA2 grid points selected for MLM input datasets (grid points only used as MLM1 inputs depicted in blue, input grid points used for MLM1 as well as MLM2 depicted in yellow, inputs used for all three models displayed in red).

Table 1. All variables used in the machine learning modeling (MLM) process and validation with their corresponding name, application, unit, size in MLM1, size in MLM2, size in MLM3 and source (in the size column, the multiplicator corresponds to the number of grid points used and the multiplicand to the time series length in hours from 2010 to 2016; 43 date dummy variables: 24-h, 7-day, and 12-month dummy variables).

\begin{tabular}{|c|c|c|c|c|c|c|}
\hline Name & Application & Unit & MLM1 Size & MLM2 Size & MLM3 Size & Source \\
\hline $\begin{array}{l}2 \mathrm{~m} \text { northward wind } \\
\text { speed }(\mathrm{V} 2 \mathrm{M})\end{array}$ & predictor & $\mathrm{m} / \mathrm{s}$ & $378 \times 61,368$ & $206 \times 61,368$ & $58 \times 61,368$ & MERRA2 \\
\hline $\begin{array}{l}10 \mathrm{~m} \text { northward wind } \\
\text { speed }(\mathrm{V} 10 \mathrm{M})\end{array}$ & predictor & $\mathrm{m} / \mathrm{s}$ & $378 \times 61,368$ & $206 \times 61,368$ & $58 \times 61,368$ & MERRA2 \\
\hline $\begin{array}{l}50 \mathrm{~m} \text { northward wind } \\
\text { speed (V50M) }\end{array}$ & predictor & $\mathrm{m} / \mathrm{s}$ & $378 \times 61,368$ & $206 \times 61,368$ & $58 \times 61,368$ & MERRA2 \\
\hline $\begin{array}{l}2 \text { m eastward wind } \\
\text { speed (U2M) }\end{array}$ & predictor & $\mathrm{m} / \mathrm{s}$ & $378 \times 61,368$ & $206 \times 61,368$ & $58 \times 61,368$ & MERRA2 \\
\hline $\begin{array}{l}10 \text { m eastward wind } \\
\text { speed (U10M) }\end{array}$ & predictor & $\mathrm{m} / \mathrm{s}$ & $378 \times 61,368$ & $206 \times 61,368$ & $58 \times 61,368$ & MERRA2 \\
\hline $\begin{array}{l}50 \text { m eastward wind } \\
\text { speed (U50M) }\end{array}$ & predictor & $\mathrm{m} / \mathrm{s}$ & $378 \times 61,368$ & $206 \times 61,368$ & $58 \times 61,368$ & MERRA2 \\
\hline date dummy variables & predictor & - & $43 \times 61,368$ & $43 \times 61,368$ & $43 \times 61,368$ & $\begin{array}{l}\text { OPSD date } \\
\text { sequence }\end{array}$ \\
\hline actual generation & response & capacity factor & 61,368 & 61,368 & 61,368 & OPSD \\
\hline RN generation & validation & capacity factor & 61,368 & 61,368 & 61,368 & Renewables.ninja \\
\hline MLM1 generation & validation & capacity factor & 61,368 & 61,368 & 61,368 & $\begin{array}{l}\text { Neural network } \\
\text { prediction }\end{array}$ \\
\hline MLM2 generation & validation & capacity factor & 61,368 & 61,368 & 61,368 & $\begin{array}{l}\text { Neural network } \\
\text { prediction }\end{array}$ \\
\hline MLM3 generation & validation & capacity factor & 61,368 & 61,368 & 61,368 & $\begin{array}{l}\text { Neural network } \\
\text { prediction }\end{array}$ \\
\hline
\end{tabular}




\subsection{Time Series Quality Assessment}

Standard model error metrics (correlations and model error) and the modeled time series' ability to replicate characteristics of the observed time series (distributions, seasonal characteristics, representation of extreme values, and power ramps) were assessed. The comparison is based on an hourly time series covering seven generation years (2010-2016). The time series quality was assessed for the whole seven-year time series. We emphasize here that we always compared the RN model to time series predicted from different trained models, i.e., we did not use training periods for comparison.

\section{Results}

In the results section, we present results of MLM1 and MLM2 in comparison to RN. The results of MLM3 are similar to MLM2 and are therefore shown only in the Appendix A.

\subsection{Model Selection}

We tested two different network sizes—one with 60 and one with 80 nodes in the hidden layers—-for generating the whole seven-year prediction time series with all considered models. The network size featuring better model error metrics was chosen for a more thorough assessment of the time series quality. When comparing error metrics for the two models MLM1 and MLM2 with a neural network of three hidden layers with 60 and 80 nodes each for the prediction period, MLM1 performs better with a smaller network size. MLM2 performs remarkably better when using the prediction dataset with the bigger network size (Table 2).

Table 2. Comparison of model error values for MLM1 and MLM2 for the training and prediction dataset.

\begin{tabular}{cccc}
\hline Error Metric & Network Size & MLM1 & MLM2 \\
\hline NMAE & 60 & 0.152 & 0.144 \\
NRMSE & 60 & 0.208 & 0.202 \\
NMAE & 80 & 0.161 & 0.138 \\
NRMSE & 80 & 0.220 & 0.190 \\
\hline
\end{tabular}

\subsection{Basic Time Series Quality}

Both MLM time series feature comparable or better error metrics than RN. MLM2 also exhibits a similarly high correlation to $\mathrm{RN}$.

The MLM1 hourly time series features a comparable NMAE (0.152), as well as a slightly lower NRMSE (0.209) value with a slightly lower hourly correlation (COR: 0.970), compared to the RN time series (NMAE: 0.152, NRMSE: 0.210, COR: 0.976). In comparison with the RN time series, the MLM2 hourly time series features a generally lower NMAE (0.138) and a lower NRMSE (0.191) value with a comparably high hourly correlation (COR: 0.975).

Both MLM1 (VAR: 0.025) and MLM2 (VAR: 0.025) estimate the total observed time series' variance (VAR: 0.026) more accurately compared to RN (VAR: 0.030). When quantiles are considered, both MLMs are closer to the observed quantiles than RN, except for the $0 \%$ quantile where the MLMs are lower due to the presence of negative values, the $25 \%$ and $100 \%$ quantile for the MLM1 time series, and the $100 \%$ quantile for the MLM2 time series. The MLM1 time series features 53 negative capacity factor events and the MLM2 features 65 (Table 3).

Except for the highest capacity factor (CF) range, both MLM time series mainly fare equally well to or better than the RN when deviations from observed values are considered. 
Table 3. Comparison of the error metrics for Renewables.ninja (RN), the first MLM-generated time series (MLM1), and the second MLM-generated time series (MLM2).

\begin{tabular}{ccccc}
\hline Quality Measure & Observations & RN & MLM1 & MLM2 \\
\hline Correlation & - & 0.976 & 0.970 & 0.975 \\
NMAE & - & 0.152 & 0.152 & 0.138 \\
NRMSE & - & 0.210 & 0.209 & 0.191 \\
Variance & 0.026 & 0.030 & 0.025 & 0.025 \\
Quantile & & & & \\
$0 \%$ & 0.000 & 0.000 & -0.012 & -0.011 \\
$25 \%$ & 0.067 & 0.071 & 0.072 & 0.069 \\
$50 \%$ & 0.137 & 0.149 & 0.140 & 0.135 \\
$75 \%$ & 0.260 & 0.275 & 0.260 & 0.254 \\
$100 \%$ & 0.913 & 0.964 & 0.970 & 0.975 \\
\hline
\end{tabular}

Median deviations in five CF ranges (except for the CF ranges of $0.0-0.1,0.4-0.5,0.5-0.6$, and above 0.8) are less distant to zero when compared with the MLM1 time series. With the MLM2 time series, the median deviation for five $\mathrm{CF}$ ranges is less distant to zero than that of the $\mathrm{RN}$ time series (except for the $\mathrm{CF}$ ranges of $0.0-0.1,0.3-0.4,0.4-0.5$, and above 0.8 ). With the $\mathrm{RN}$ time series, an overestimation is apparent in the scatterplot, particularly in the higher CF spectrum. The ranges of deviations for MLM1 are slightly wider than those of the $\mathrm{RN}$ time series, except for the values of $0.0-0.1,0.1-0.2$, and $0.2-0.3$. In a similar way, the deviation ranges of MLM2 are only narrower than the RN deviations in two CF classes (CF 0.0-0.1 and CF 0.1-0.2). The deviation median in the MLM2 time series is generally less distant to zero than in the MLM1 time series, except for the CF classes of $0.2-0.3$ and 0.3-0.4. For the deviation ranges, the picture is fairly the same, with the MLM2 time series featuring a narrower range in five CF ranges $(0.0-0.1,0.4-0.5,0.5-0.6,0.6-0.7$, and $0.7-0.8)$. This is also reflected in the scatterplot with the MLM2 values more concentrated towards the diagonal line than the MLM1 values. Both MLM time series also mainly underestimate generation slightly within most CF classes, whereas the RN time series overestimates generation (Figure 5).

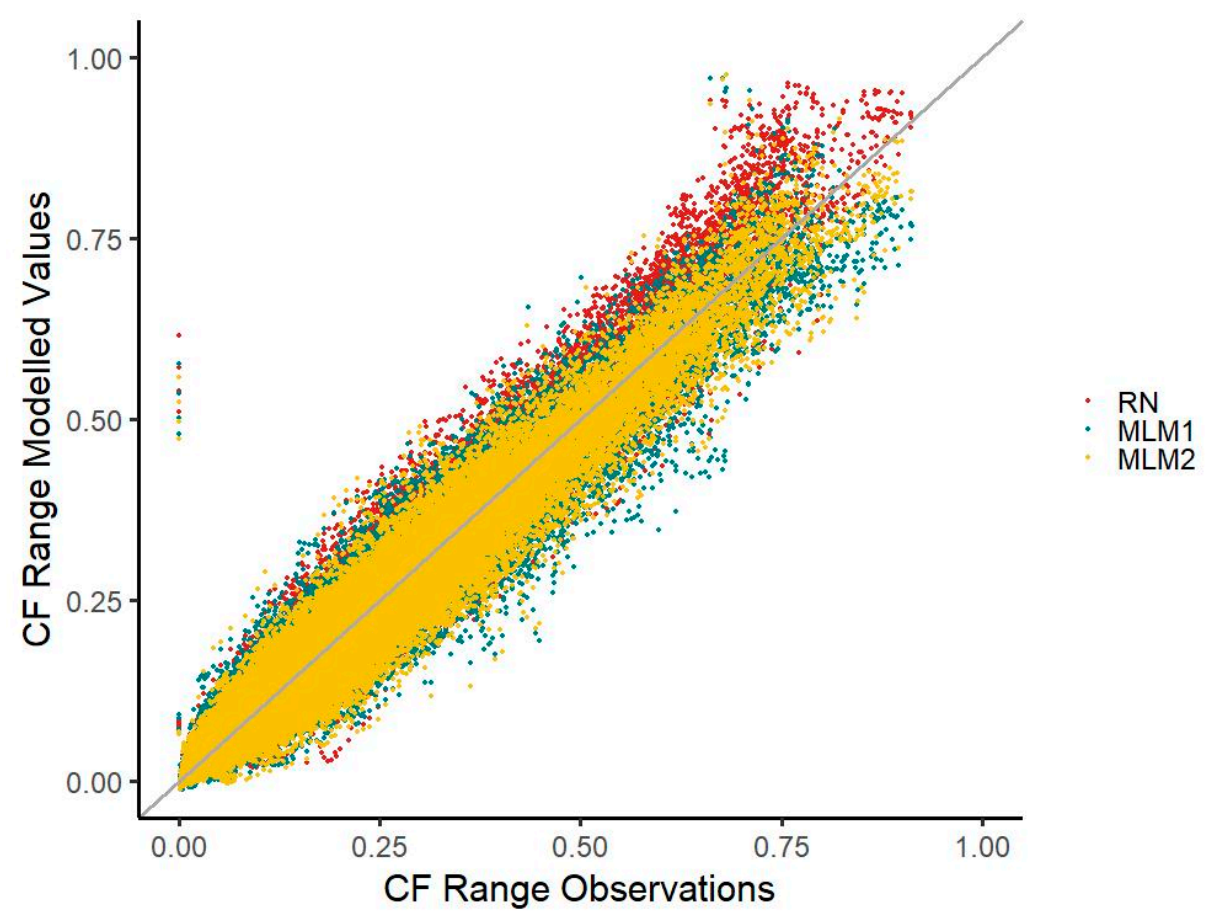

Figure 5. Scatterplot of modeled generation time series compared with observations (Renewables.ninja time series abbreviated as RN and machine learning model time series abbreviated as MLM1 and MLM2 versus observations on the $X$-axis). 
The MLM1 and MLM2 time series reduce the underestimation of CF values around 0.1 visible in the RN time series. However, both MLMs underestimate the occurrences of CFs in the very low range compared with RN.

The results show an acceptable representation of the distributional characteristics of the observed time series comparable to the results of RN for both MLM time series. Generation in the very low CF range ( 8254 actual values with a CF $<0.04)$ is better approximated by the RN time series (8138 modeled values with a CF < 0.04) than by MLM1 (6658 modeled values) or MLM2 (7115 modeled values) time series, which is also represented by a higher density in the $\mathrm{RN}$ time series. CFs in the range around 0.1 (8928 actual values between 0.08 and 0.12 ), where an overestimation occurs in the MLM-derived time series (9146 modeled values for the MLM1 time series and 9249 for MLM2) opposed to an underestimation in the RN time series (7989 modeled values), however, are better represented by the MLMs. This can also be seen by a better fit of the probability density curve in this range for both MLMs. In the high CF range, the frequency of CF values above 0.8 is, although slightly underestimated (75 and 126 values $>0.8$ for the MLM1 and MLM2 versus 121 actual values), better approximated by the MLM time series than by RN (471 modeled values $>0.8$ ) (Figure 6).

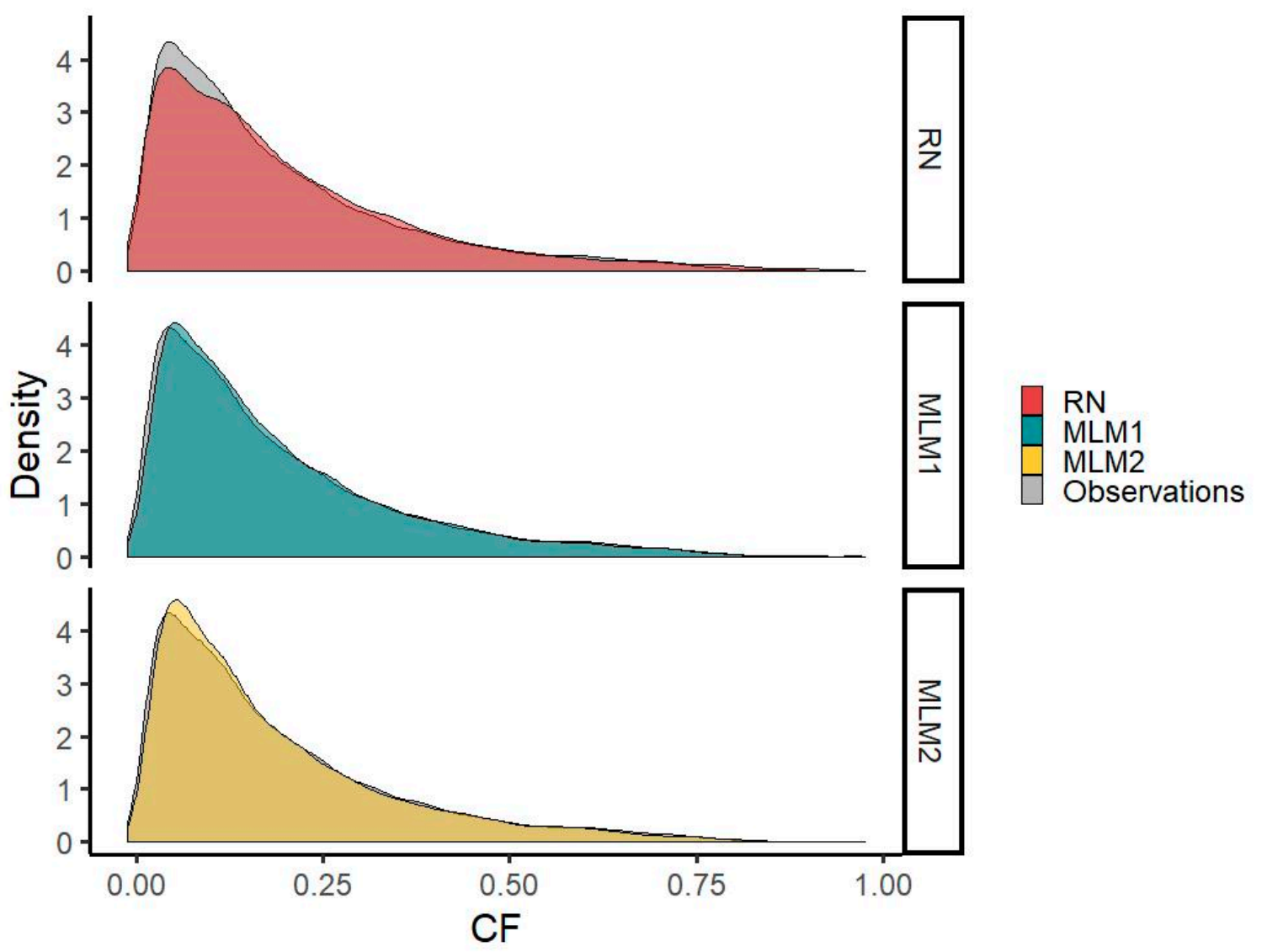

Figure 6. Total distributions of the RN, MLM1, and MLM2 modeled time series compared with the observed total distribution (dark colored areas correspond to an accordance of the modeled and observed time series; Renewables.ninja time series abbreviated as RN and machine learning model time series abbreviated as MLM1 and MLM2).

\subsection{Diurnal Characteristics}

MLM2 provides a better estimate of diurnal characteristics for most hours and both MLM time series reduce deviations in the evening hours featured in the RN time series (Figure 7).

RN median deviations are generally lower than those of MLM1, except for seven hours (hours 2, $15,16,17,18,19$, and 20). For MLM2, the median deviations are generally lower than those of the RN 
time series, except for four hours (hours 4, 6, 22, and 23). For RN, the mean of deviations is highest around the evening (hours 18,19, and 20), where it is noticeably higher than during the rest of the day. It is lowest during the morning (hours 7 and 8) and around midnight (hours 1 and 24), where it is lower than during the remaining hours. The MLM1 and MLM2 time series do not feature a similarly strong increase of the deviation median in the evening hours.

The deviation range of the MLM1 time series is narrower for $8 \mathrm{~h}$ (hours 1, 2, 3, 4, 5, 6, 22, and 23) compared with the RN time series, and for $12 \mathrm{~h}$ (hours 1, 2, 3, 4, 5, 6, 7, 9, 21, 22, 23, and 24) when compared with the MLM2 time series. For all three time series, the deviation range is remarkably wider in the early morning hours $2-5$ than during the remaining hours. With all three time series (most remarkably with the MLM1 time series), an increase of the deviation range around noon and early afternoon can be seen and for all three time series, the deviation range decreases again for the evening hours (Figure 7).

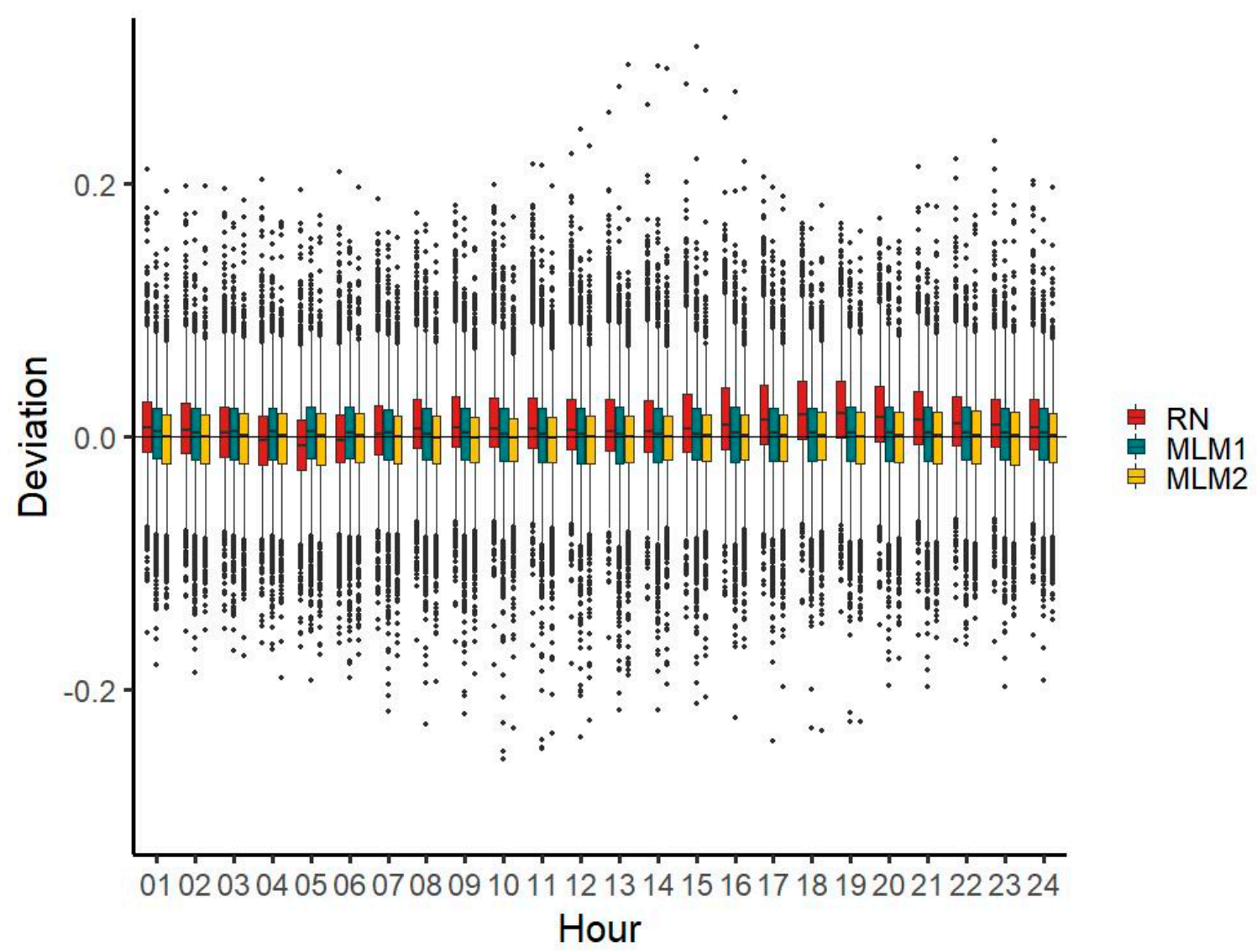

Figure 7. Hourly deviations from observed values for RN, MLM1, and MLM2 (Renewables.ninja time series abbreviated as RN and machine learning model time series abbreviated as MLM1 and MLM2).

\subsection{Seasonal Characteristics}

Here, we split all three time series into an autumn-winter and a spring-summer half-year to compare seasonal characteristics. March, April, May, June, July, and August constitute the spring-summer half year, and the remaining months comprise the autumn-winter half-year. RN, MLM1, and MLM2 reflect seasonal characteristics comparably well (Figure 8). However, MLM2 performs better than MLM1 in the majority of CF classes. Interestingly, the lowest CF class in the autumn-winter half-year features four outliers in all three time series.

When comparing observations made with the MLM1 time series, the median deviation is lower than for RN for three out of 10 CF classes, where MLM1 is better in the CF classes 0.0-0.2, 0.2-0.4, and $0.6-0.8$ in the autumn-winter half-year. The MLM2 time series performs better in four out of 
10 CF classes-the same classes as MLM1-and in the 0.6-0.8 class for the spring-summer half-year. This means that the RN time series fares better than both MLM time series in a seasonal comparison; however, for most classes, only by a small margin. For all three time series, a tendency to overestimate values in the lowest CF class can be observed. In the remaining CF classes, the MLM time series mainly underestimate, whereas the RN time series overestimates. In the comparison of the deviation ranges (spread from the minimum to maximum deviation value), MLM2 fares equally well as MLM1, outperforming the RN time series in four out of 10 classes. Both MLMs perform better than RN in the class $0.0-0.2$ in both half-years, in the $0.6-0.8$ class for the autumn-winter half-year, and in the $>0.8$ class for the spring-summer half-year. Remarkably, the lowest class in the autumn-winter half-year features some outliers, skewing all three time series towards a high deviation range. For the deviation mean, MLM2 outperforms RN in more CF classes than MLM1 (Figure 8).

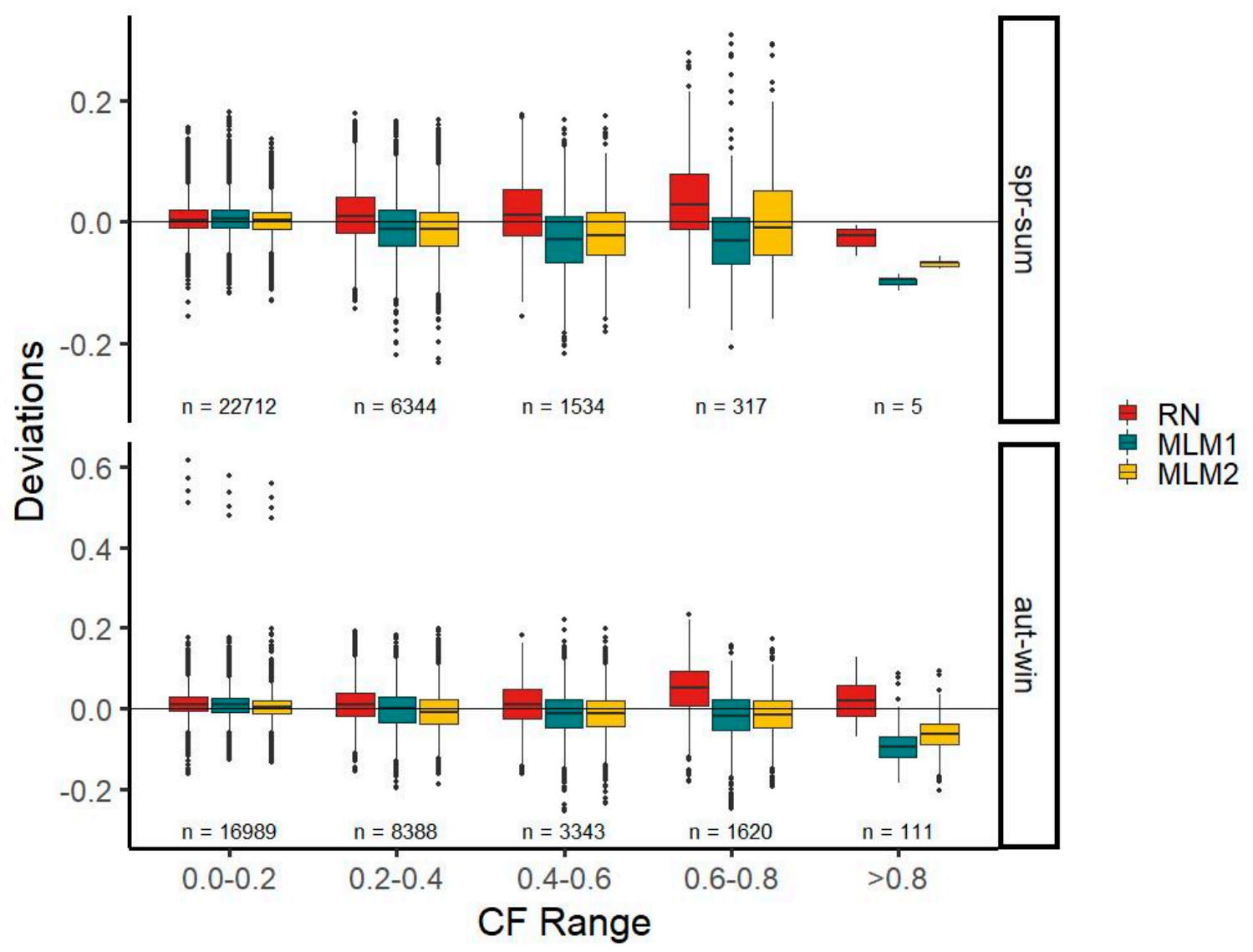

Figure 8. Capacity factor (CF) deviations of RN, MLM1, and MLM2 modeled time series within different CF bins for the autumn-winter and spring-summer half year from 2010 to 2016 (Renewables.ninja time series abbreviated as RN, machine learning model time series abbreviated as MLM1 and MLM2, and n denotes the number of observations within CF bins).

\subsection{Durations and Frequencies of Low, High, and Extreme Values}

MLM1 and MLM2 provide a better estimate of frequencies and durations of low CF extremes and frequencies of high CF extremes. Durations of high CF extremes are better approximated by RN.

Frequencies (102 actual events) of low-capacity factor extreme values $(\mathrm{CF}<0.005)$ are better approximated by MLM1 (193 modeled events) and MLM2 (238 modeled events) than by the RN time series (430 events), where both MLMs provide a less pronounced overestimation and therefore better approximation. The mean durations (3.19 consecutive hours of observed generation below $0.005 \mathrm{CF}$ ) of the low-capacity factor extreme values are also better approximated by MLM1 (3.27 consecutive hours of modeled generation below $0.005 \mathrm{CF}$ ) and MLM2 (3.50 consecutive hours of modeled generation below 
$0.005 \mathrm{CF}$ ) compared with RN (4.62 consecutive hours of modeled generation below $0.005 \mathrm{CF}$ ). Both MLM time series provide an exact match of the low generation extreme maximum duration (10 observed consecutive hours below $0.005 \mathrm{CF}$ ), whereas the $\mathrm{RN}$ time series overestimates the maximum duration (14 consecutive hours of modeled generation below 0.005 CF). Frequencies (121 actual events) of high-capacity factor extreme values (CF $>0.8$ ) are better approximated by MLM1 (75 modeled events) and MLM2 (125 modeled events) than by RN (471 modeled events). When mean durations of very high generation events (7.56 observed consecutive hours) are considered, RN (9.24 modeled consecutive hours) provides the best estimate compared with MLM1 (4.17 modeled consecutive hours) and MLM2 (5.21 modeled consecutive hours). With regard to the approximation of maximum durations (35 consecutive observed hours), the MLM1 time series (11 modeled consecutive hours) is fairly far off, whereas RN (33 modeled consecutive hours) and MLM2 (21 modeled consecutive hours) provide better estimates (Table 4).

Table 4. Observed and modeled (Renewables.ninja [RN] and machine learning models [MLM1 and MLM2]) CF, including frequencies, mean, and maximum durations of extremely low $(<0.005)$, low $(0.01)$, high (0.75), and extremely high ( $>0.8)$ generation events in consecutive hours.

\begin{tabular}{ccccc}
\hline CF Ranges & Observations & RN & MLM1 & MLM2 \\
\hline Frequency & CF $<0.005$ & & & \\
Mean Duration & 102 & 430 & 193 & 238 \\
Max Duration & 3.19 & 4.62 & 3.27 & 3.50 \\
& 10 & 14 & 10 & 10 \\
Frequency & CF $<0.01$ & & & \\
Mean Duration & 509 & 748 & 312 & 341 \\
Max Duration & 2.98 & 2.60 & 1.91 & 1.91 \\
& 25 & 12 & 7 & 5 \\
Frequency & $\mathrm{CF}>0.75$ & & & \\
Mean Duration & 204 & 356 & 174 & 225 \\
Max Duration & 3.46 & 3.10 & 3.63 & 3.41 \\
& 13 & 18 & 22 & 15 \\
Frequency & $\mathrm{CF}>0.8$ & & & \\
Mean Duration & 121 & 471 & 75 & 125 \\
Max Duration & 7.56 & 9.24 & 4.17 & 5.21 \\
\hline
\end{tabular}

\subsection{Frequencies and Ranges of Power Ramps}

Both MLM time series follow the cumulative density of capacity factor changes within one hour better than the RN time series. In particular, the RN time series overestimates the density within the range of negative $\mathrm{CF}$ changes between -0.1 and 0 and underestimates it within the range of positive CF changes between 0 and 0.1. MLM1 and MLM2 do not show this behavior and nearly match the observed CF change density exactly (Figure 9).

Within all considered time frames, both MLM time series replicate CF changes better than or equally well to the RN time series. CF changes within four different time frames $(1,3,6$, and $12 \mathrm{~h})$ are better than or equally well replicated with MLMs compared to RN. Both MLM time series feature mean values of positive and negative $C F$ changes, frequencies of negative and positive $C F$ changes, minimum and maximum ramp values, and an approximation of the frequencies of fairly high and low power ramps comparable to or better than the $\mathrm{RN}$ within nearly all time frames. All models fare better the longer the time frame, reflecting the ability of the reanalysis data source to replicate the temporal variability of wind speeds within longer time frames (Table 5). 


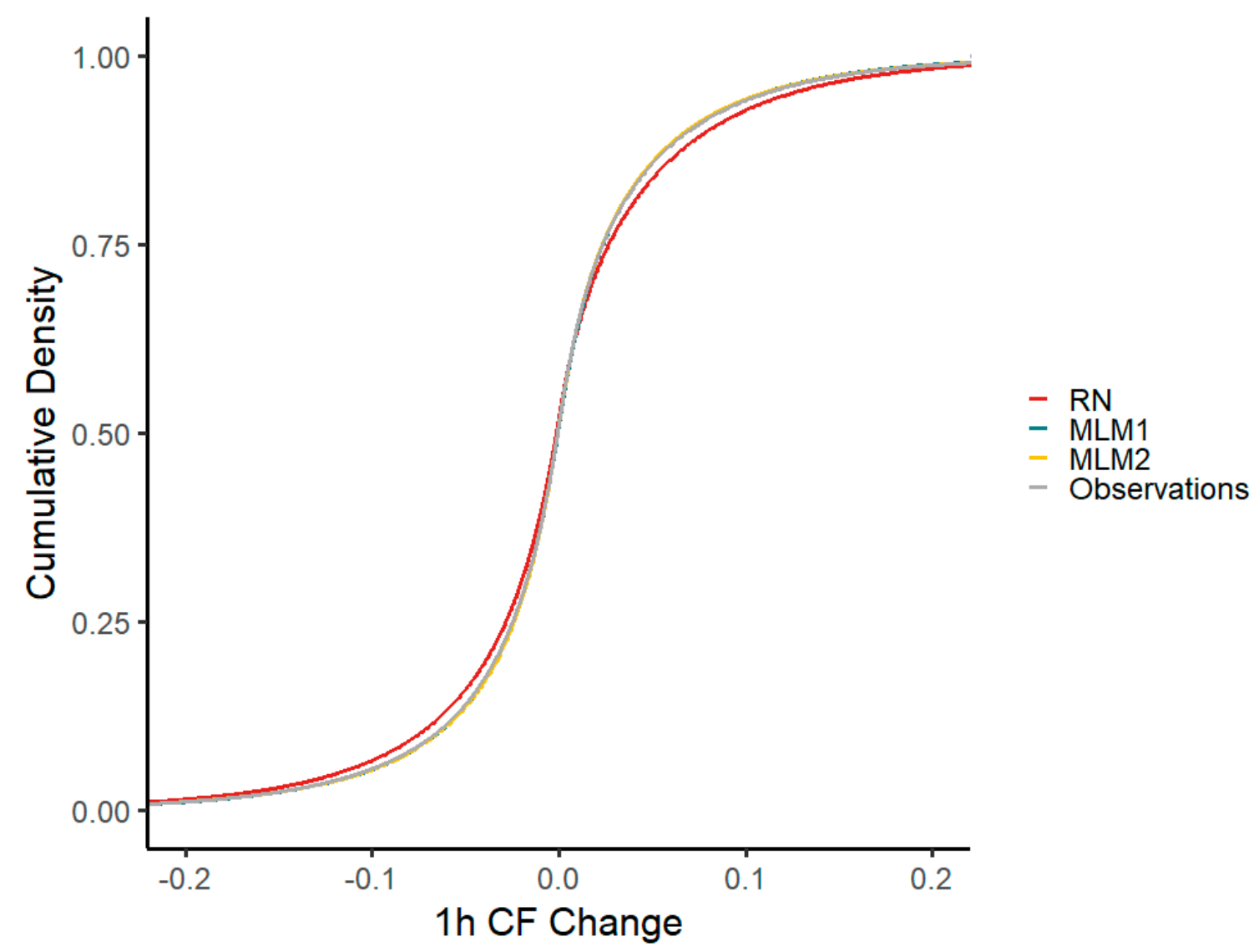

Figure 9. Cumulative density of one-hour capacity factor (CF) changes for the RN, MLM1, and MLM2 modeled time series compared with observations.

Table 5. Minimum, maximum, and mean of negative and positive capacity factor (CF) ramps within different time frames and frequency of highly positive and negative CF ramps.

\begin{tabular}{ccccc}
\hline Time Frame & Observations & RN & MLM1 & MLM2 \\
\hline $1 \mathrm{~h}$ & & & & \\
Min & -0.66 & -0.11 & -0.14 & -0.13 \\
Neg. Mean & -0.012 & -0.013 & -0.013 & -0.012 \\
Neg. Freq & 31,313 & 32,243 & 31,070 & 31,132 \\
Pos. Mean & 0.013 & 0.014 & 0.013 & 0.013 \\
Pos. Freq & 30,054 & 29,124 & 30,297 & 30,235 \\
Max & 0.50 & 0.16 & 0.16 & 0.14 \\
Frequency $<-0.2$ & 1 & 0 & 0 & 0 \\
Frequency $>0.2$ & 1 & 0 & 0 & 0 \\
3 h & & & & \\
Min & -0.67 & -0.28 & -0.30 & -0.33 \\
Neg. Mean & -0.022 & -0.024 & -0.023 & -0.022 \\
Neg. Freq & 94,293 & 96,677 & 93,587 & 94,091 \\
Pos. Mean & 0.023 & 0.027 & 0.023 & 0.023 \\
Pos. Freq & 89,805 & 87,421 & 90,411 & 90,007 \\
Max & 0.50 & 0.30 & 0.38 & 0.31 \\
Frequency $<-0.2$ & 75 & 71 & 84 & 61 \\
Frequency $>0.2$ & 92 & 181 & 85 & 93 \\
\hline
\end{tabular}


Table 5. Cont.

\begin{tabular}{ccccc}
\hline Time Frame & Observations & RN & MLM1 & MLM2 \\
\hline 6h & & & & \\
Min & -0.67 & -0.46 & -0.48 & -0.50 \\
Neg. Mean & -0.035 & -0.038 & -0.035 & -0.034 \\
Neg. Freq & 188,949 & 192,660 & 188,102 & 188,749 \\
Pos. Mean & 0.037 & 0.042 & 0.036 & 0.036 \\
Pos. Freq & 179,238 & 175,527 & 180,085 & 179,438 \\
Max & 0.51 & 0.50 & 0.55 & 0.47 \\
Frequency $<-0.2$ & 1616 & 2140 & 1678 & 1736 \\
Frequency $>0.2$ & 1846 & 2921 & 1650 & 1860 \\
12 h & & & & \\
Min & -0.68 & -0.60 & -0.66 & -0.64 \\
Neg. Mean & -0.054 & -0.059 & -0.053 & -0.053 \\
Neg. Freq & 376,200 & 380,753 & 375,262 & 376,127 \\
Pos. Mean & 0.056 & 0.064 & 0.055 & 0.055 \\
Pos. Freq & 360,138 & 355,585 & 361,076 & 360,211 \\
Max & 0.62 & 0.67 & 0.65 & 0.68 \\
Frequency $<-0.2$ & 14,185 & 18,009 & 13,781 & 14,245 \\
Frequency $>0.2$ & 14,680 & 19,194 & 13,634 & 14,237 \\
\hline
\end{tabular}

\section{Discussions}

The MLM approach can only be successfully applied if (1) sufficiently long and high-quality climate input data and generation data are available for the prevailing wind conditions and turbine locations in a region, which is currently not the case for all regions. This, however, does not translate to a downside of using a neural network approach as this also holds true for power curve-based models, which need observations for calibration or validation. (2) The MLMs are not capable of reflecting changes in the spatial configuration of the installed wind turbine capacity as installed capacities are not used as model inputs. However, the proposed approach can be easily adapted to make use of information on installed capacities. (3) The applicability of wind power models is quite diverse. Therefore, the scope of this study was limited to a comparison of the time series quality for RN and the proposed MLM approach. The advantages and disadvantages of using one method over the other can be different, depending on the application; however, a large difference in impact between using a power curve-based or MLM-derived one is not to be expected based on the comparison of time series quality. The proposed approach of deriving time series by means of neural networks is only (4) partly suited to generating future scenarios taking significant technological developments into account (e.g., a considerable shift of the ratio between the rotor diameter and installed capacity, such as in turbines specifically designed for low wind speed conditions) compared with a power curve-based approach such as in RN. Using turbine specifications as an input to model training can probably compensate for this downside in subsequent model iterations, although this would require additional technical information on the turbine types used. (5) Landmark changes in technology (e.g., horizontal turbine design) or the regulation of wind turbines or intermittent renewables (e.g., increased curtailment) in general, for which no observational data are available, cannot be successfully replicated by the proposed neural network approach. This issue can be addressed for both methodologies using post treatment of the model output. (6) For the MLM training step, a significant amount of computational effort is required, which is potentially higher than the computational effort associated with the model setup of power curve-based models. The prediction step, however, is comparable in computational complexity to power curve-based approaches.

\section{Conclusions}

All three machine learning models were able to generate wind power generation time series comparable to or even better than a state-of-the-art power curve-based modeling approach 
(Renewables.ninja, abbreviated as RN) with respect to standard error metrics, seasonal and distributional characteristics, and frequencies and durations of low, high, and extreme values, as well as for the replication of frequencies and durations of power ramps for wind power generation.

We used three datasets-one without location information, and two with implicit location information via incremental climate data grid point subsetting-as the input for MLMs to assess whether location information is needed to obtain a time series quality comparable to that of RN. We found that (1) all three input datasets for the machine learning model time series were able to generate wind power generation time series comparable to or even better than a state-of-the-art power curve-based modeling approach (RN) with respect to the quality measures considered. (2) Furthermore, the information required for model setup with regard to knowing accurate wind turbine locations and power curves is much lower. The additional information on turbine locations and the used turbine models is not strictly needed to reach a time series quality comparable to that of the RN approach, although implicit location information improves most of the time series quality measures considered as MLM2 and MLM3 outperform MLM1 in most cases. This translates to less spatial information on installed capacities being required when using the ML-based approach, while still attaining a time series quality similar to RN. (3) All MLM-generated time series (especially MLM2 and MLM3) additionally show a reduced overestimation of very high $C F$ values and reduced underestimation of $C F$ values around a CF of 0.1 . (4) The time series quality varies, depending on which quality parameter is considered and on which MLM is used. However, there are no major drawbacks of using a machine learning approach for the purpose of generating wind power time series, with the exception of the duration of high generation extreme events, which are rare. (5) The presence of negative CF values indicates that longer training periods could be helpful as the whole range of all input variable combinations and thus, the variability of climate data has not been fully captured by the available training time frames. However, negative values can easily be handled via simple post-processing.

Author Contributions: Conceptualization, J.B. and J.S.; methodology, J.B., K.G., and J.S.; software, J.B.; validation, J.B., K.G., and J.S.; formal analysis, J.B. and K.G.; investigation, J.B.; resources, J.S.; data curation, J.B.; writing-original draft preparation, J.B.; writing-review and editing, J.B., K.G., S.-D.Y.M., J.S., and S.G.S.; visualization, J.B.; supervision, J.S.; project administration, J.S. and S.G.S.; funding acquisition, Y.-M.S.-D., J.S., and S.G.S. All authors have read and agreed to the published version of the manuscript.

Funding: We acknowledge funding from CLIM2POWER. Project CLIM2POWER is part of ERA4CS, an ERA-NET initiated by JPI Climate, and funded by FORMAS (SE), BMBF (DE), BMWFW (AT), FCT (PT), EPA (IE), and ANR (FR) with co-funding by the European Union (Grant 690462). We also gratefully acknowledge support from the European Research Council ("reFUEL" ERC-2017-STG 758149).

Acknowledgments: We would like to cordially thank Iain Staffell for the provision of historic observed generation data used for Renewables.ninja model calibration. Open access funding provided by BOKU Vienna Open Access Publishing Fund.

Conflicts of Interest: The authors declare no conflicts of interest. The funders had no role in the design of the study; in the collection, analyses, or interpretation of data; in the writing of the manuscript; or in the decision to publish the results.

\section{Appendix A}

\section{Appendix A.1. Results of a Model with a Stronger Subsetting of the Climate Data Grid Points (MLM3)}

For this model, we reduced the number of climate data grid points even further. Installed capacities were attributed to the nearest grid point based on wind farm locations given in OPSD [44]. Only sites with an installed capacity above the third quartile were used (58 grid points used versus 206 used in MLM2). This is only possible when not only wind farm locations are known, but also their installed capacities, which translates into a higher degree of required location information.

When testing two different network sizes-one with 60 and one with 80 nodes in the hidden layers-for generating the whole seven-year prediction time series with MLM3, we found MLM3 performing slightly better with the smaller network size (Table A1). 
Table A1. Comparison of model error values for all tested models for the training and prediction dataset.

\begin{tabular}{ccccc}
\hline Error Metric & Network Size & MLM1 & MLM2 & MLM3 \\
\hline NMAE & 60 & 0.152 & 0.144 & 0.138 \\
NRMSE & 60 & 0.208 & 0.202 & 0.194 \\
NMAE & 80 & 0.161 & 0.138 & 0.141 \\
NRMSE & 80 & 0.220 & 0.190 & 0.194 \\
\hline
\end{tabular}

\section{Appendix A.2. Basic Time Series Quality of MLM3}

The MLM3 hourly time series features a similarly high correlation (0.975), a similarly low NMAE (0.138), and only a slightly higher NRMSE (0.194) value compared with the MLM2 time series (NMAE: 0.138, NRMSE: 0.194, COR: 0.975).

The total observed time series' variance (VAR: 0.026) was estimated to be only slightly less accurate with MLM3 (0.024) than with MLM2 (VAR: 0.025). When quantiles are considered, the MLM3 time series performs better for three quartiles $(0 \%, 50 \%$, and $100 \%)$ when compared to MLM2. The MLM3 time series exhibits 81 negative capacity factor events compared with 65 for MLM2 (Table A2).

Table A2. Comparison of error metrics for RN, MLM1, and MLM2 approaches.

\begin{tabular}{ccccc}
\hline Quality Measure & Observations & RN & MLM2 & MLM3 \\
\hline Correlation & - & 0.976 & 0.975 & 0.975 \\
NMAE & - & 0.152 & 0.138 & 0.138 \\
NRMSE & - & 0.210 & 0.191 & 0.194 \\
Variance & 0.026 & 0.030 & 0.025 & 0.024 \\
Quantile & & & & \\
$0 \%$ & 0.000 & 0.000 & -0.011 & -0.008 \\
$25 \%$ & 0.067 & 0.071 & 0.069 & 0.070 \\
$50 \%$ & 0.137 & 0.149 & 0.135 & 0.136 \\
$75 \%$ & 0.260 & 0.275 & 0.254 & 0.251 \\
$100 \%$ & 0.913 & 0.964 & 0.975 & 0.943 \\
\hline
\end{tabular}

Median deviations in two capacity factor (CF) ranges (0.1-0.2 and 0.6-0.7) are less distant to zero when compared with the MLM2 time series, which translates into an average underestimation that is stronger than that of MLM2. The ranges of deviations for MLM3 are slightly narrower than those of MLM2 time series for values of $0.0-0.1,0.3-0.4,0.4-0.5$, and $>0.8$. Both time series look fairly similar in a scatterplot (Figure A1).

The distributional characteristics of the MLM3 time series are similar to those of the MLM2 time series. Generation frequencies in the very low $\mathrm{CF}$ range $(8254$ actual values with a $\mathrm{CF}<0.04$ ) are better approximated by the MLM2 time series $(7115$ modeled values with a CF $<0.04)$ than by MLM3 (6859 modeled values). CF frequencies in the mid-range from around 0.2 to 0.5 (17,772 actual values with a CF between 0.2 and 0.5 ) are also slightly better approximated by the MLM2 time series (17,397 modeled values between 0.2 and 0.5$)$ than by MLM3 (17,240 modeled values with a CF between 0.2 and 0.5 ). In the range from 0.1 to 0.2 , higher densities can be seen with the MLM3 time series. The same holds true for the range around 0.1 (8928 actual values between 0.08 and 0.12 ), where the MLM3 time series (9300 modeled values) overestimates slightly more than the MLM2 time series (9249 modeled values), although this is not easily visible when comparing densities. In the high CF range (121 observed values $>0.8$ ), the MLM3 (115 modeled values) underestimates and the MLM2 (126 modeled values) overestimates (Figure A2). 


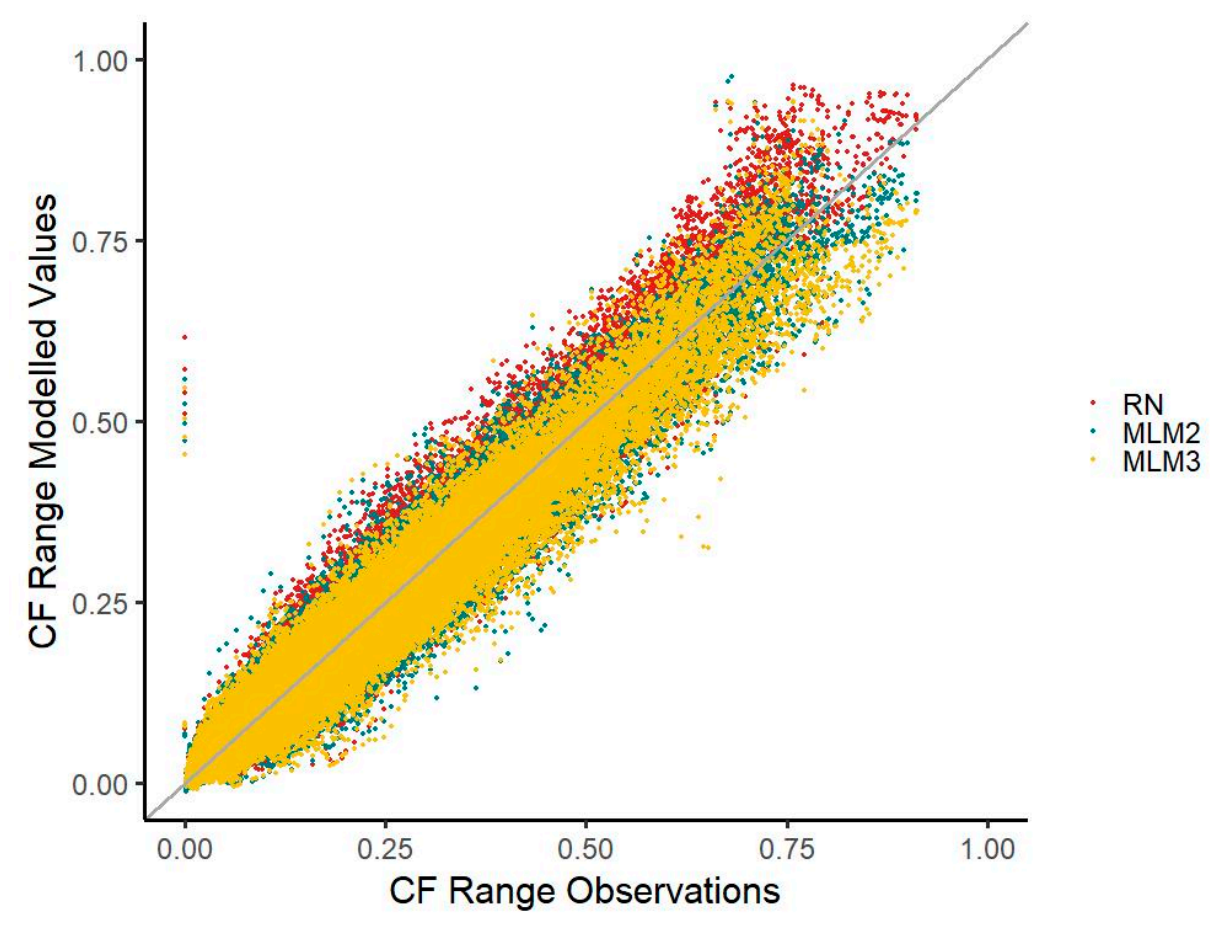

Figure A1. Scatterplot of modeled generation time series compared with observations (Renewables.ninja time series abbreviated as RN and machine learning model time series abbreviated as MLM2 and MLM3 versus observations on the $X$-axis).

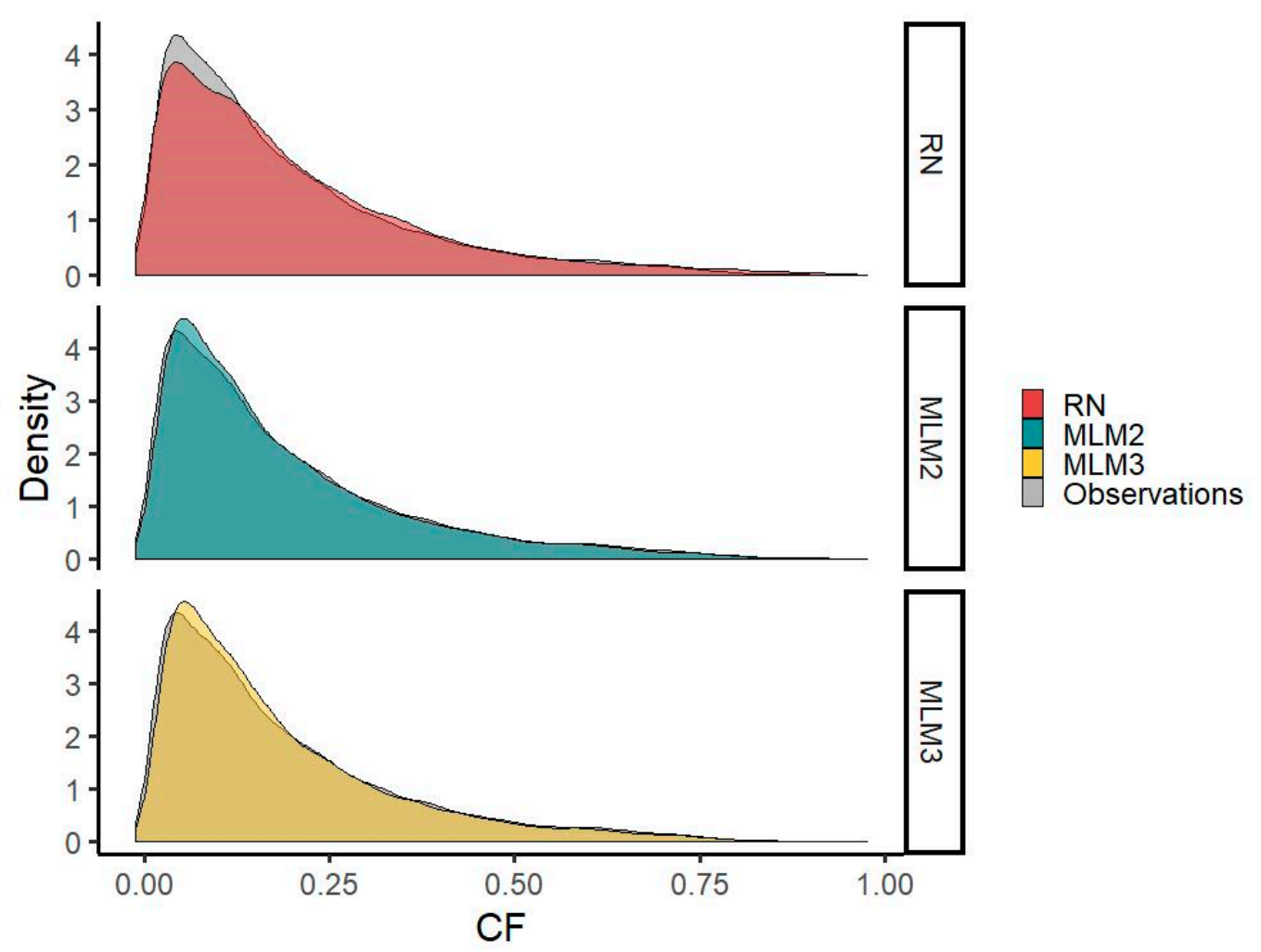

Figure A2. Total distributions of the RN, MLM2, and MLM3 modeled time series compared with the observed total distribution (dark colored areas correspond to an accordance of the modeled and observed time series; Renewables.ninja time series abbreviated as RN and machine learning model time series abbreviated as MLM2 and MLM3). 


\section{Appendix A.3. Diurnal Characteristics of MLM3}

The MLM3 median deviations are lower than those of MLM2 in the evening and night (hours 17-24) and morning (hours 1-8) h. Around noon (hours 9-14), the MLM2 median deviations are lower.

The differences in the deviation range between the MLM3 and MLM2 time series do not follow the same patterns as with median deviations, with MLM3 featuring a narrower deviation range for eight morning and evening hours (hours $3,4,5,8,10,17,18$, and 19) (Figure A3).

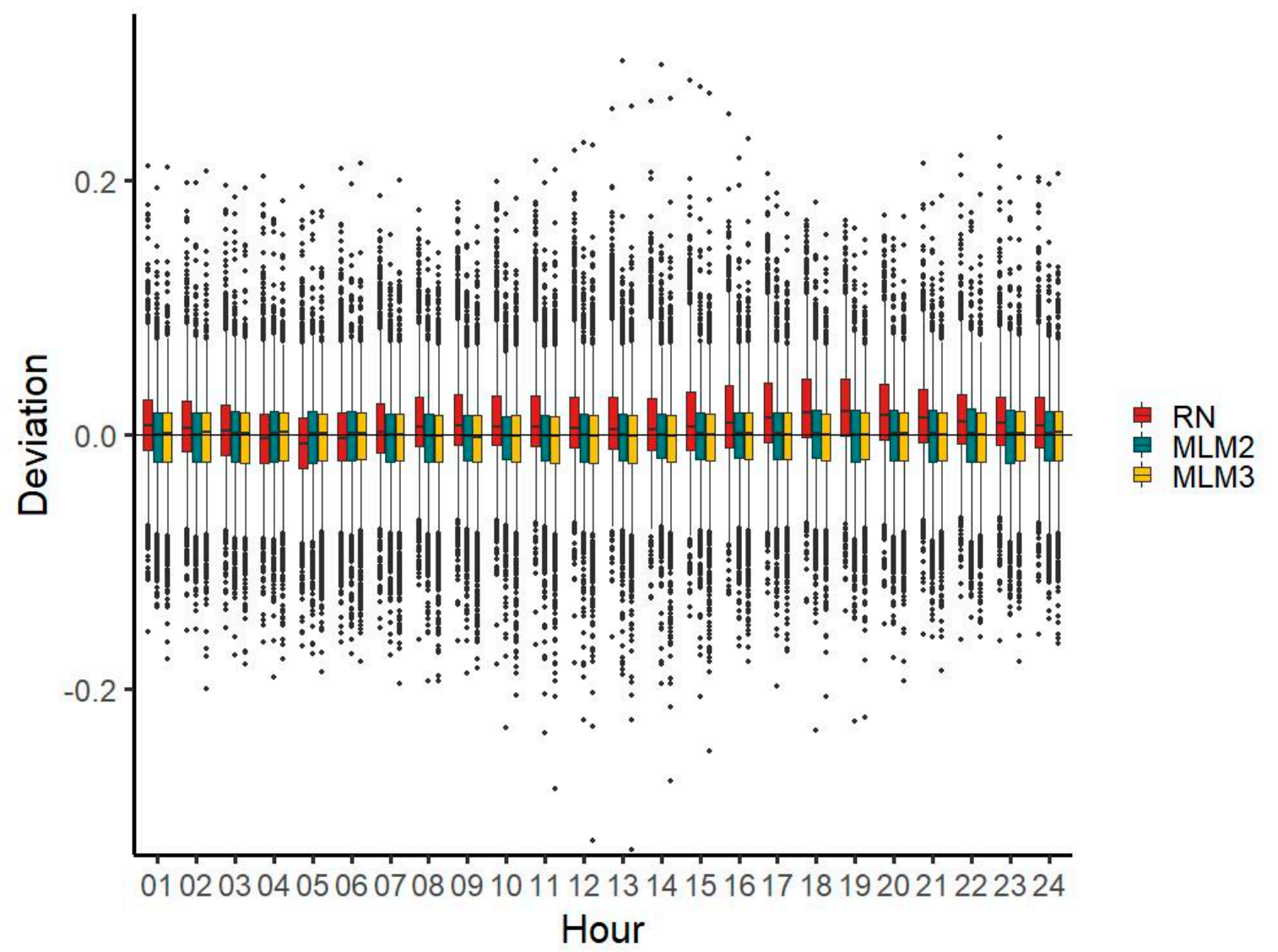

Figure A3. Hourly deviations from observed values for RN, MLM2, and MLM3 (Renewables.ninja time series abbreviated as RN and machine learning model time series abbreviated as MLM2 and MLM3).

Appendix A.4. Seasonal Characteristics of MLM3

MLM3 outperforms RN in three out of 10 CF classes. Compared with MLM2, the median deviation for MLM3 is only lower in the 0.6-0.8 class for the spring-summer half-year. The MLM2 time series performs better than RN in four out of $10 \mathrm{CF}$ classes. The MLM3 deviation range is lower than that of MLM2 in four classes, although it only has a lower deviation range than RN in three classes. For both ML time series, a tendency to underestimate events in all classes except the CF class $0.0-0.2$ can be seen (Figure A4). 


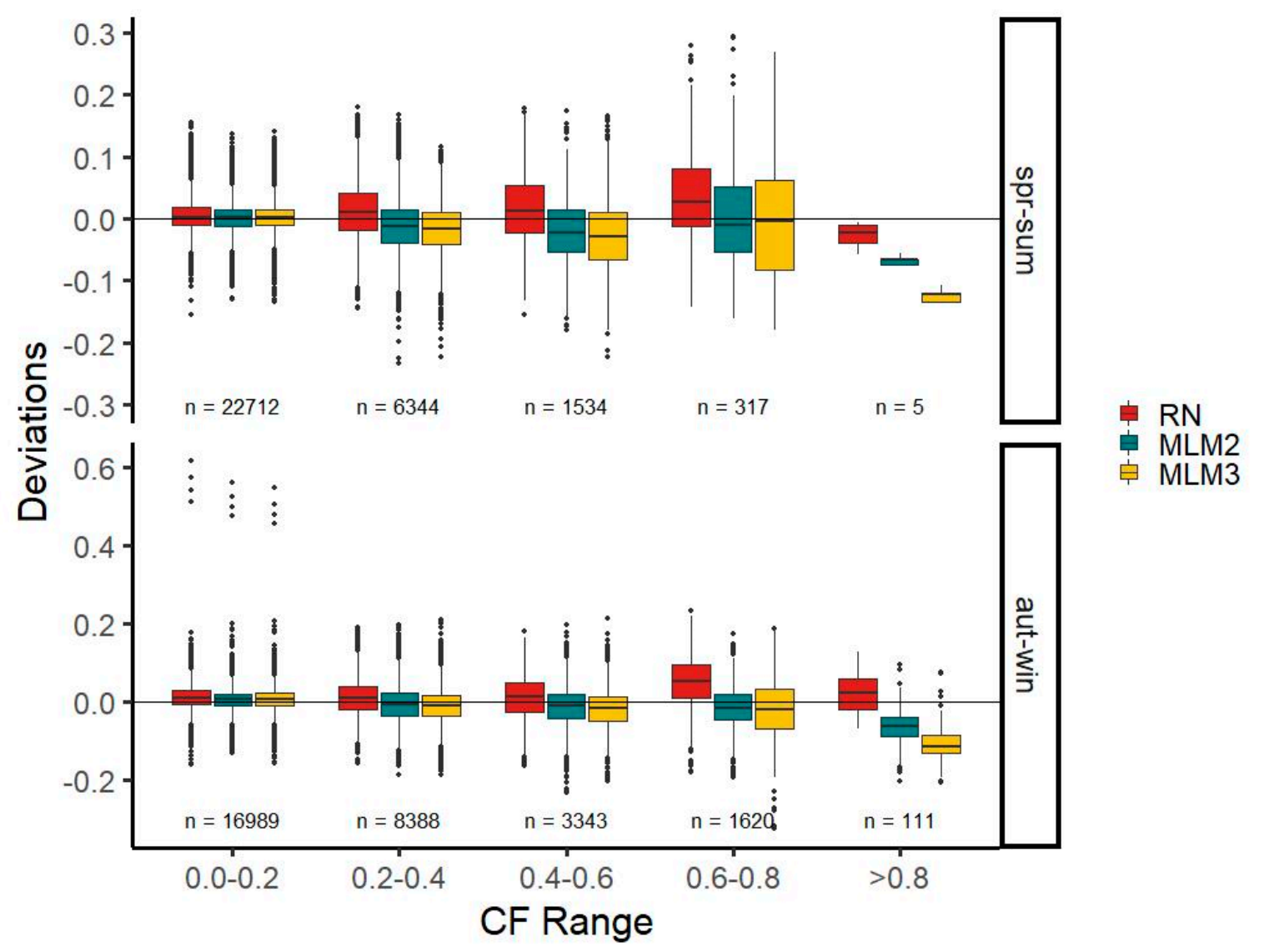

Figure A4. Capacity factor (CF) deviations of RN, MLM2, and MLM3 modeled time series within different CF bins for the autumn-winter and spring-summer half-year from 2010 to 2016 (Renewables.ninja time series abbreviated as RN, machine learning model time series abbreviated as MLM2 and MLM3, and n denotes the number of observations within CF bins).

Appendix A.5. Durations and Frequencies of Low, High, and Extreme Values of MLM3

Frequencies of low-capacity factor extreme values $(\mathrm{CF}<0.005)$ are better approximated by MLM2 (238 modeled events) than by MLM3 (248 modeled events). Mean durations of consecutive CFs below 0.005 are also better approximated by MLM2 (3.50 modelled hours of observed generation below $0.005 \mathrm{CF}$ ) compared with MLM3 (3.70 consecutive hours of modeled generation below $0.005 \mathrm{CF}$ ). The same holds true where low generation extreme maximum durations are concerned, whereas the MLM3 time series overestimates the maximum duration (15 consecutive hours of modeled generation below $0.005 \mathrm{CF})$. Frequencies of high-capacity factor extreme values ( $\mathrm{CF}>0.8$ ) are better approximated by MLM2 (125 modeled events) than by MLM3 (115 modeled events). Mean durations of very high generation events, however, are better approximated by MLM3 (7.19 modeled consecutive hours) than by MLM2 (5.21 modeled consecutive hours). Taking the maximum duration into account, MLM2 (21 modeled consecutive hours) provides a better estimate than MLM3 (17 modeled consecutive hours) (Table A3).

Appendix A.6. Frequencies and Ranges of Power Ramps of MLM3

For the shorter time frames ( 1 and $3 \mathrm{~h}$ ), MLM3 performs better than MLM2, with CF change means and frequencies being approximated better than or equal to the MLM2 time series; however, MLM2 performs better the longer the time frames (Table A4). 
Table A3. Observed and modeled (Renewables.ninja [RN] and machine learning models [MLM2 and MLM3]) CF, including frequencies, mean, and maximum durations of extremely low $(<0.005)$, low (0.01), high (0.75), and extremely high (>0.8) generation events in consecutive hours.

\begin{tabular}{ccccc}
\hline CF Ranges & Observations & RN & MLM2 & MLM3 \\
\hline Frequency & CF $<0.005$ & & & \\
Mean Duration & 102 & 430 & 238 & 248 \\
Max Duration & 3.19 & 4.62 & 3.50 & 3.70 \\
& 10 & 14 & 10 & 15 \\
Frequency & CF $<0.01$ & & & \\
Mean Duration & 509 & 748 & 341 & 283 \\
Max Duration & 2.98 & 2.60 & 1.91 & 1.78 \\
Frequency & 25 & 12 & 5 & 7 \\
Mean Duration & CF $>0.75$ & & & \\
Max Duration & 204 & 356 & 225 & 187 \\
& 3.46 & 3.10 & 3.41 & 3.46 \\
Frequency & 13 & 18 & 15 & 21 \\
Mean Duration & $\mathrm{CF}>0.8$ & & & \\
Max Duration & 121 & 471 & 125 & 115 \\
\hline
\end{tabular}

Table A4. Minimum, maximum, and mean of negative and positive capacity factor (CF) ramps within different time frames and frequency of highly positive and negative CF ramps.

\begin{tabular}{|c|c|c|c|c|}
\hline Time Frame & Observations & $\mathbf{R N}$ & MLM2 & MLM3 \\
\hline \multicolumn{5}{|l|}{$1 \mathrm{~h}$} \\
\hline Min & -0.66 & -0.11 & -0.13 & -0.11 \\
\hline Neg. Mean & -0.012 & -0.013 & -0.012 & -0.012 \\
\hline Neg. Freq & 31,313 & 32,243 & 31,132 & 31,308 \\
\hline Pos. Mean & 0.013 & 0.014 & 0.013 & 0.012 \\
\hline Pos. Freq & 30,054 & 29,124 & 30,235 & 30,059 \\
\hline $\operatorname{Max}$ & 0.50 & 0.16 & 0.14 & 0.15 \\
\hline Frequency $<-0.2$ & 1 & 0 & 0 & 0 \\
\hline $\begin{array}{c}\text { Frequency }>0.2 \\
3 \mathrm{~h}\end{array}$ & 1 & 0 & 0 & 0 \\
\hline Min & -0.67 & -0.28 & -0.33 & -0.30 \\
\hline Neg. Mean & -0.022 & -0.024 & -0.022 & -0.022 \\
\hline Neg. Freq & 94,293 & 96,677 & 94,091 & 94,389 \\
\hline Pos. Mean & 0.023 & 0.027 & 0.023 & 0.023 \\
\hline Pos. Freq & 89,805 & 87,421 & 90,007 & 89,709 \\
\hline $\operatorname{Max}$ & 0.50 & 0.30 & 0.31 & 0.32 \\
\hline Frequency $<-0.2$ & 75 & 71 & 61 & 69 \\
\hline $\begin{array}{c}\text { Frequency }>0.2 \\
6 \mathrm{~h}\end{array}$ & 92 & 181 & 93 & 79 \\
\hline Min & -0.67 & -0.46 & -0.50 & -0.47 \\
\hline Neg. Mean & -0.035 & -0.038 & -0.034 & -0.034 \\
\hline Neg. Freq & 188,949 & 192,660 & 188,749 & 189,180 \\
\hline Pos. Mean & 0.037 & 0.042 & 0.036 & 0.036 \\
\hline Pos. Freq & 179,238 & 175,527 & 179,438 & 179,007 \\
\hline $\operatorname{Max}$ & 0.51 & 0.50 & 0.47 & 0.47 \\
\hline Frequency $<-0.2$ & 1616 & 2140 & 1736 & 1682 \\
\hline $\begin{array}{c}\text { Frequency }>0.2 \\
12 \mathrm{~h}\end{array}$ & 1846 & 2921 & 1860 & 1687 \\
\hline Min & -0.68 & -0.60 & -0.64 & -0.62 \\
\hline Neg. Mean & -0.054 & -0.059 & -0.053 & -0.052 \\
\hline Neg. Freq & 376,200 & 380,753 & 376,127 & 377,137 \\
\hline Pos. Mean & 0.056 & 0.064 & 0.055 & 0.055 \\
\hline Pos. Freq & 360,138 & 355,585 & 360,211 & 359,201 \\
\hline $\operatorname{Max}$ & 0.62 & 0.67 & 0.68 & 0.63 \\
\hline Frequency $<-0.2$ & 14,185 & 18,009 & 14,245 & 13,396 \\
\hline Frequency $>0.2$ & 14,680 & 19,194 & 14,237 & 13,580 \\
\hline
\end{tabular}




\section{References}

1. Maaßen, U. Arbeitsgemeinschaft Energiebilanzen Bruttostromerzeugung in Deutschland ab 1990 nach Energieträgern. Available online: https://ag-energiebilanzen.de/index.php?article_id=29\&fileName= 20181214_brd_stromerzeugung1990-2018.pdf (accessed on 4 May 2020).

2. Global Wind Energy Council. Global Wind Report 2018; Global Wind Energy Council: Brussels, Belgium, 2019.

3. Global Wind Energy Council. Global Wind 2007 Report; Global Wind Energy Council: Brussels, Belgium, 2008.

4. WindEurope. Wind Energy in Europe in 2018 Trends and Statistics; WindEurope: Brussels, Belgium, 2019.

5. Stanton, M.B.; Dessai, S.; Paavola, J. A systematic review of the impacts of climate variability and change on electricity systems in Europe. Energy 2016, 109, 1148-1159. [CrossRef]

6. Klein, D.R.; Olonscheck, M.; Walther, C.; Kropp, J.P. Susceptibility of the European electricity sector to climate change. Energy 2013, 59, 183-193. [CrossRef]

7. Tobin, I.; Greuell, W.; Jerez, S.; Ludwig, F.; Vautard, R.; Van Vliet, M.T.H.; Bréon, F.-M.; Van Vliet, M.T.H. Vulnerabilities and resilience of European power generation to $1.5{ }^{\circ} \mathrm{C}, 2{ }^{\circ} \mathrm{C}$ and $3{ }^{\circ} \mathrm{C}$ warming. Environ. Res. Lett. 2018, 13, 044024. [CrossRef]

8. Réseau de Transport D'électricité Shedding Light on the Future of the Energy System. Available online: https://antares-simulator.org/ (accessed on 1 September 2019).

9. Strbac, G.; Aunedi, M.; Papadaskalopoulos, D.; Qadrdan, M.; Moreno, R.; Pudjianto, D.; Djapic, P.; Konstantelos, I.; Teng, F. Modelling of Smart Low-Carbon Energy Systems; wholeSEM: London, UK, 2014.

10. E3M Manual of PRIMES. Available online: http://www.e3mlab.eu/e3mlab/index.php?option=com_content\& view=article\&id=58\%3Amanual-for-primes-model\&catid=35\%3Aprimes\&Itemid=80\&lang=en (accessed on 1 September 2019).

11. Loulou, R.; Goldstein, G.; Kanudia, A.; Lettila, A.; Remme, U. Documentation for the TIMES Model-Part I; IEA: Paris, France, 2016.

12. Simoes, S.; Zeyringer, M.; Mayr, D.; Huld, T.; Nijs, W.; Schmidt, J. Impact of different levels of geographical disaggregation of wind and PV electricity generation in large energy system models: A case study for Austria. Renew. Energy 2017, 105, 183-198. [CrossRef]

13. Staffell, I.; Pfenninger, S. Using bias-corrected reanalysis to simulate current and future wind power output. Energy 2016, 114, 1224-1239. [CrossRef]

14. Andresen, G.; Søndergaard, A.A.; Greiner, M. Validation of Danish wind time series from a new global renewable energy atlas for energy system analysis. Energy 2015, 93, 1074-1088. [CrossRef]

15. Cannon, D.; Brayshaw, D.; Methven, J.; Coker, P.; Lenaghan, D. Using reanalysis data to quantify extreme wind power generation statistics: A 33 year case study in Great Britain. Renew. Energy 2015, 75, 767-778. [CrossRef]

16. Gruber, K.; Klöckl, C.; Regner, P.; Baumgartner, J.; Schmidt, J. Assessing the Global Wind Atlas and local measurements for bias correction of wind power generation simulated from MERRA-2 in Brazil. Energy 2019, 189, 116212. [CrossRef]

17. Staffell, I.; Green, R. How does wind farm performance decline with age? Renew. Energy 2014, 66, 775-786. [CrossRef]

18. Zucker, A.; Gonzalez Aparicio, I.; Careri, F.; Monforti, F.; Huld, T.; Badger, J.; European Commission. Joint Research Centre EMHIRES Dataset Part I, Wind Power Generation: European Meteorological Derived HIgh Resolution RES Generation Time Series for Present and Future Scenarios; Publications Office: Luxembourg, 2016.

19. Olauson, J.; Bergkvist, M. Modelling the Swedish wind power production using MERRA reanalysis data. Renew. Energy 2015, 76, 717-725. [CrossRef]

20. Cybenko, G. Approximation by superpositions of a sigmoidal function. Math. Control. Signals Syst. 1989, 2, 303-314. [CrossRef]

21. Moraes, L.; Bussar, C.; Stoecker, P.; Jacqué, K.; Chang, M.; Sauer, D.U. Comparison of long-term wind and photovoltaic power capacity factor datasets with open-license. Appl. Energy 2018, 225, 209-220. [CrossRef]

22. Grams, C.M.; Beerli, R.; Pfenninger, S.; Staffell, I.; Wernli, H. Balancing Europe's wind-power output through spatial deployment informed by weather regimes. Nat. Clim. Chang. 2017, 7, 557-562. [CrossRef] [PubMed]

23. Collins, S.; Deane, P.; Gallachóir, B.Ó.; Pfenninger, S.; Staffell, I. Impacts of Inter-annual Wind and Solar Variations on the European Power System. Joule 2018, 2, 2076-2090. [CrossRef] [PubMed] 
24. Badami, M.; Fambri, G.; Mancò, S.; Martino, M.; Damousis, I.; Agtzidis, D.; Tzovaras, D. A Decision Support System Tool to Manage the Flexibility in Renewable Energy-Based Power Systems. Energies 2019, 13, 153. [CrossRef]

25. Chang, G.W.; Lu, H.; Chang, Y.; Lee, Y. An improved neural network-based approach for short-term wind speed and power forecast. Renew. Energy 2017, 105, 301-311. [CrossRef]

26. Heinermann, J.; Kramer, O. Machine learning ensembles for wind power prediction. Renew. Energy 2016, 89, 671-679. [CrossRef]

27. Khosravi, A.; Koury, R.; Machado, L.; García-Pabón, J.J. Prediction of wind speed and wind direction using artificial neural network, support vector regression and adaptive neuro-fuzzy inference system. Sustain. Energy Technol. Assess. 2018, 25, 146-160. [CrossRef]

28. Treiber, N.A.; Heinermann, J.; Kramer, O. Wind Power Prediction with Machine Learning. In Applications of Hybrid Metaheuristic Algorithms for Image Processing; Springer Science and Business Media LLC: Berlin, Germany, 2016; Volume 645, pp. 13-29.

29. Aghajani, A.; Kazemzadeh, R.; Ebrahimi, A. A novel hybrid approach for predicting wind farm power production based on wavelet transform, hybrid neural networks and imperialist competitive algorithm. Energy Convers. Manag. 2016, 121, 232-240. [CrossRef]

30. Dong, Q.; Sun, Y.; Li, P. A novel forecasting model based on a hybrid processing strategy and an optimized local linear fuzzy neural network to make wind power forecasting: A case study of wind farms in China. Renew. Energy 2017, 102, 241-257. [CrossRef]

31. Esfetang, N.N.; Kazemzadeh, R.; Esfetanaj, N.N. A novel hybrid technique for prediction of electric power generation in wind farms based on WIPSO, neural network and wavelet transform. Energy 2018, 149, 662-674. [CrossRef]

32. Park, J.; Park, J. Physics-induced graph neural network: An application to wind-farm power estimation. Energy 2019, 187, 115883. [CrossRef]

33. Gelaro, R.; Mccarty, W.; Suárez, M.J.; Todling, R.; Molod, A.; Takacs, L.; Randles, C.; Darmenov, A.; Bosilovich, M.G.; Reichle, R.H.; et al. The Modern-Era Retrospective Analysis for Research and Applications, Version 2 (MERRA-2). J. Clim. 2017, 30, 5419-5454. [CrossRef] [PubMed]

34. Downloads-Renewables.ninja. Available online: https://www.renewables.ninja/downloads (accessed on 28 June 2018).

35. Abhinav, R.; Pindoriya, N.M.; Wu, J.; Long, C. Short-term wind power forecasting using wavelet-based neural network. Energy Procedia 2017, 142, 455-460. [CrossRef]

36. Díaz, S.; Carta, J.; Matías, J.M. Performance assessment of five MCP models proposed for the estimation of long-term wind turbine power outputs at a target site using three machine learning techniques. Appl. Energy 2018, 209, 455-477. [CrossRef]

37. Foley, A.M.; Leahy, P.G.; Marvuglia, A.; McKeogh, E. Current methods and advances in forecasting of wind power generation. Renew. Energy 2012, 37, 1-8. [CrossRef]

38. Data Platform-Open Power System Data. Available online: https://data.open-power-system-data.org/time_ series/ (accessed on 28 June 2018).

39. Bergmeir, C.; Benitez, J.M. Neural Networks in R Using the Stuttgart Neural Network Simulator: RSNNS. J. Stat. Softw. 2012, 46, 46. [CrossRef]

40. Kuhn, M. Building Predictive Models in R Using the caret Package. J. Stat. Softw. 2008, 28. [CrossRef]

41. Schmidt, J. MERRAbin. Available online: https://github.com/joph/MERRAbin (accessed on 28 October 2019).

42. Baumgartner, J. Machine-Learning-Long-Term-Wind-Power-Time-Series. Available online: https://github. com/jbaumg/Machine-learning-long-term-wind-power-time-series (accessed on 29 November 2019).

43. Baumgartner, J. Machine-Learning-Long-Term-Wind-Power-Time-Series. Available online: https://zenodo. org/record/3784661 (accessed on 4 May 2020).

44. Data Platform-Open Power System Data. Available online: https://data.open-power-system-data.org/ renewable_power_plants/ (accessed on 16 August 2018).

(C) 2020 by the authors. Licensee MDPI, Basel, Switzerland. This article is an open access article distributed under the terms and conditions of the Creative Commons Attribution (CC BY) license (http://creativecommons.org/licenses/by/4.0/). 